\title{
Szenarien und Projektionen zur weltweiten Energieversorgung im Vergleich
}

\author{
Hans-Wilhelm Schiffer ${ }^{1,2}$ \\ Online publiziert: 14. August 2020 \\ ๑) Springer Fachmedien Wiesbaden GmbH, ein Teil von Springer Nature 2020
}

\section{Zusammenfassung}

Szenarien und Prognosen sind ein unverzichtbares „Werkzeug“ für die Entscheidungsfindung der Politik ebenso wie zur Ausrichtung der Strategie von Unternehmen. Angesichts der langfristigen Auswirkungen von Investitionen gilt dies in besonderer Weise für die Energieversorgung. Das vorliegende Papier richtet den Blick auf die weltweiten Perspektiven. Dazu haben verschiedene Institutionen Ausarbeitungen vorgelegt, in denen Angebot und Nachfrage aller Energien und Technologien berücksichtigt sind. In jüngerer Zeit waren dies vor allem die Internationale Energie-Agentur (IEA), die U.S. Energy Information Administration (EIA), Shell, ExxonMobil, BP, Equinor, DNV-GL, McKinsey und der World Energy Council (WEC). Trotz bestehender Differenzen in Methodik und Erkenntnis lassen sich deutliche Gemeinsamkeiten in der Bewertung der zukünftigen globalen Energieversorgung erkennen. Die Aussagen richten sich auf die bevorstehenden Jahrzehnte, teilweise bis zum Jahr 2040 oder sogar deutlich darüber hinaus. Die Schlüsselbotschaften, die aus den Studien der genannten Organisationen ableitbar sind, werden dargelegt. Ferner werden mögliche Auswirkungen der COVID-19-Pandemie, die in den analysierten Arbeiten noch nicht berïcksichtigt sind, thematisiert.

Schlüsselwörter Szenarien und Prognosen · Globale Energieversorgung · Wandel im Energiemix · Digitalisierung ·

Dekarbonisierung $\cdot$ Elektrifizierung $\cdot$ Wasserstoff-Strategie

\section{Synopsis of Scenarios and Projections of the Global Energy Supply}

\begin{abstract}
Scenarios and forecasts are an indispensable "tool" for decision-making in politics as well as for aligning the strategy of companies. In view of the long-term effects of investments, this applies in particular to the energy supply. This paper focuses on global perspectives. For this purpose, various institutions have submitted elaborations in which the supply and demand of all energies and technologies are taken into account. More recently, these have been primarily the International Energy Agency (IEA), the U.S. Energy Information Administration (EIA), Shell, ExxonMobil, BP, Equinor, DNV-GL, McKinsey and the World Energy Council (WEC). Despite existing differences in methodology and knowledge, there are clear similarities in the assessment of future global energy supplies. The statements refer to the coming decades, in some cases up to 2040 or even significantly beyond. The key messages that can be derived from the studies of the organizations mentioned are presented. Possible effects of the COVID-19 pandemic, which have not yet been considered in the analyzed work, are also discussed.
\end{abstract}

Keywords Scenarios and forecasts · Global energy supply · Change in the energy mix · Digitalization · Decarbonization · Electrification - Hydrogen strategy

Dr. Hans-Wilhelm Schiffer

hwschiffer@t-online.de

1 Silkestr. 25, 50999 Köln, Deutschland

2 Fakultät für Georessourcen und Materialtechnik - Lehr- und Forschungsgebiet Technologie der Energierohstoffe, RWTH Aachen, Aachen, Deutschland 


\section{Einleitung}

Für eine Bewertung der in Zukunftsaussagen zur Energieversorgung ausgewiesenen Ergebnisse sind der zugrunde gelegte methodische Ansatz und die getroffenen Annahmen von besonderer Relevanz. Grundsätzlich kann zwischen exploratorischen Szenarien, Projektionen und normativen Szenarien unterschieden werden (World Energy Council 2019a). In exploratorischen Szenarien wird aufgezeigt, wohin unterstellte Entwicklungen unter Berücksichtigung der gesetzten Grundannahmen führen würden. Es handelt sich nicht um Vorhersagen. Eintrittswahrscheinlichkeiten sind exploratorischen Szenarien nicht zugeordnet. Im Vordergrund steht ein Narrativ, das modellgestützt quantitativ unterlegt wird. Bei Projektionen gibt es unterschiedliche Ausprägungen. So wird in Prognosen versucht, die künftige Entwicklung auf Basis von als wahrscheinlich angenommenen Parametern unter anderem zur Entwicklung der Demografie, der Wirtschaftsleistung, technologischen Innovationen, der Weltmarktpreise für Energie und erwarteter politischer Rahmensetzung darzulegen. Prognosen zielen also darauf, die für wahrscheinlich gehaltene Entwicklung ab- zubilden. Davon zu unterscheiden sind Projektionen, die aufzeigen, was voraussichtlich passiert, wenn beispielsweise die bestehenden gesetzlichen Grundlagen künftig unverändert bleiben oder wenn - alternativ zu diesem Ansatz - auch sämtliche Maßnahmen umgesetzt werden, die von den Regierungen angekündigt sind, ohne dass sie bereits in Kraft gesetzt wurden. Einen ganz anderen Charakter haben normative Szenarien. Sie geben Ziele vor und zeigen auf, was passieren müsste, damit die für einen künftigen Zeitpunkt definierten Vorgaben erreicht werden. Die vorliegende Arbeit konzentriert sich auf Studien zu den Perspektiven der weltweiten Energieversorgung, die sowohl konventionelle als auch erneuerbare Energieträger einbeziehen und mit Veröffentlichung im Jahr 2019 den jeweils aktuellsten Stand der Analysen widerspiegeln, die von den berücksichtigten Institutionen veröffentlicht wurden.

\section{Einordnung der erfassten Studien}

Die Zukunftspfade, die in den analysierten Studien beschrieben werden, fügen sich unterschiedlich in die skiz-

\section{Szenarien und Projektionen verschiedener Institutionen zur Entwicklung der globalen Energieversorgung}

\begin{tabular}{|c|c|c|c|c|}
\hline & Organisation/Studie & $\begin{array}{l}\text { Exploratorische } \\
\text { (Plausible) Szenarien }\end{array}$ & Projektionen & Normative Szenarien \\
\hline \multirow[t]{2}{*}{$\begin{array}{c}\text { WORLD } \\
\text { ENERGY } \\
\text { COUNCILI }\end{array}$} & $\begin{array}{l}\text { WEC (2019) World Energy } \\
\text { Scenarios } 2019 \text { (to 2060) }\end{array}$ & $\begin{array}{l}\text { - Modern Jazz (MJ) } \\
\text { - Unfinished Symphony } \\
\text { (US) } \\
\text { - Hard Rock (HR) } \\
\end{array}$ & & \\
\hline & $\begin{array}{l}\text { Shell }(2013 / 18) \text { New Lens } \\
\text { Scenarios Mountain Ocean } \\
\text { (2013) and Sky (2018) } \\
\text { to } 2100\end{array}$ & $\begin{array}{l}\text { - Mountain (M) } \\
\text { - Ocean }(O)\end{array}$ & & - Sky (S) \\
\hline equinor & $\begin{array}{l}\text { Equinor (2019) Energy } \\
\text { Perspectives to } 2050\end{array}$ & $\begin{array}{l}\text { - Reform (Rf) } \\
\text { - Rivalry (Rv) } \\
\end{array}$ & & - Renewal (Rn) \\
\hline \multirow[t]{3}{*}{ eia } & $\begin{array}{l}\text { EIA (2019) International } \\
\text { Energy Outlook } 2019 \\
\end{array}$ & & - Reference Case (RC) & \\
\hline & $\begin{array}{l}\text { IEA (2019) World Energy } \\
\text { Outlook } 2019 \text { (to 2040) } \\
\end{array}$ & & \begin{tabular}{|l|} 
- Current policies (CP) \\
- Stated Policies (SP) \\
\end{tabular} & $\begin{array}{l}\text { - Sustainable } \\
\text { Development (SD) }\end{array}$ \\
\hline & $\begin{array}{l}\text { BP (2019) Energy Outlook } \\
\text { to } 2040^{\mathrm{a}}\end{array}$ & & - Evolving Transition (ET) & - Rapid Transition (RT) \\
\hline \multirow[t]{2}{*}{ EXON } & $\begin{array}{l}\text { ExxonMobil } 2019 \text { Outlook } \\
\text { for Energy: A Perspective to } 20\end{array}$ & & - Reference & \\
\hline & $\begin{array}{l}\text { DNV GL (2019) Energy } \\
\text { Transition Outlook to } 2050\end{array}$ & & $\begin{array}{l}\text { - A single forecast of the } \\
\text { energy future }\end{array}$ & \\
\hline $\begin{array}{l}\text { Mckinsey } \\
\text { \& Company }\end{array}$ & Mc Kinsey (2019) & & - Reference Case (RC) & \\
\hline
\end{tabular}

Abb. 1 Szenarien und Projektionen verschiedener Institutionen zur Entwicklung der globalen Energieversorgung. "Zusätzlich sind in der BP-Studie „Alternative“ Szenarien ausgewiesen, wie u. a. „More Energy, Less Globalization“ 
zierten Cluster ein (Abb. 1). Mit den nachfolgenden Ausführungen wird dies verdeutlicht.

\subsection{Projektionen von ExxonMobil, DNV-GL, McKinsey und EIA}

Einige der Studien lassen sich durch Einordnung in die Kategorie Projektionen einem einzigen der gebildeten Cluster zuordnen. Dabei handelt es sich um die Ausarbeitungen von ExxonMobil, DNV-GL, McKinsey und der U.S. Energy Information Administration (EIA).

Der Outlook for Energy: A Perspective to 2040 von ExxonMobil basiert zur Abschätzung der künftigen EnergieVersorgungsstrukturen auf firmenintern entwickelten Modellen. In die Rechnungen gehen Annahmen zu einer Vielzahl von Variablen ein, wie unter anderem zur Verbesserung der Energieeffizienz, zur Geschwindigkeit in der Umsetzung von neuen Technologien und von Innovationen, zu den erwarteten Trends bei den Kosten verschiedener Technologie-Optionen, zur Entwicklung von Verbraucherpräferenzen, zur politischen Rahmensetzung sowie zur geopolitischen und wirtschaftlichen Entwicklung. DNV-GL, ein norwegischer Klassifizierungs- und Zertifizierungskonzern mit weltweiten Aktivitäten im maritimen Öl-, Gas- und Energiebereich, hat mit dem Energy Transition Outlook (ETO) mittels einer eigenständigen Modellierung eine Vorhersa- ge zur Entwicklung von Energieangebot und -nachfrage bis 2050 sowohl global als auch für zehn Weltregionen vorgelegt. Basis der Vorausschätzung ist das Energy Transition Outlook Model (ETOM) - ein dynamisches Simulationsmodell, das die Beziehungen zwischen Nachfrage und Angebot in verschiedenen miteinander verknüpften Modulen reflektiert. Der ETO zielt darauf, eine Vorhersage zur wahrscheinlichen Entwicklung als Grundlage für Strategieprozesse von Unternehmen und anderen Institutionen zu liefern. McKinsey stützt sich mit seiner Studie Global Energy Perspective (2019) auf Beiträge von hunderten Experten aus seinem globalen Netzwerk, deren Expertise die ganze Palette der im Bereich der Energieversorgung wichtigen Felder umfasst. Auf diese Weise wird ein konsensualer Referenzfall gebildet, der die Sichtweise dieses Beratungsunternehmens auf die globale Ausrichtung der Energieversorgung bis 2050 abbildet.

Während die Arbeiten von ExxonMobil, DNL-GV und McKinsey demnach als Prognosen angesehen werden können, hat der Reference Case (RC) der U.S. Energy Information Administration (EIA) einen anderen Charakter. So ist der RC der EIA als Baseline-Einschätzung anzusehen, die sich auf den Fortbestand der derzeit gültigen politischen und regulatorischen Rahmenbedingungen - etwa hinsichtlich der eingegangenen Verpflichtungen von Regierungen zum Klimaschutz - über den gesamten Betrachtungszeit-

\section{Globaler Primärenergieverbrauch 1979 bis 2019}

\section{in Exajoules}

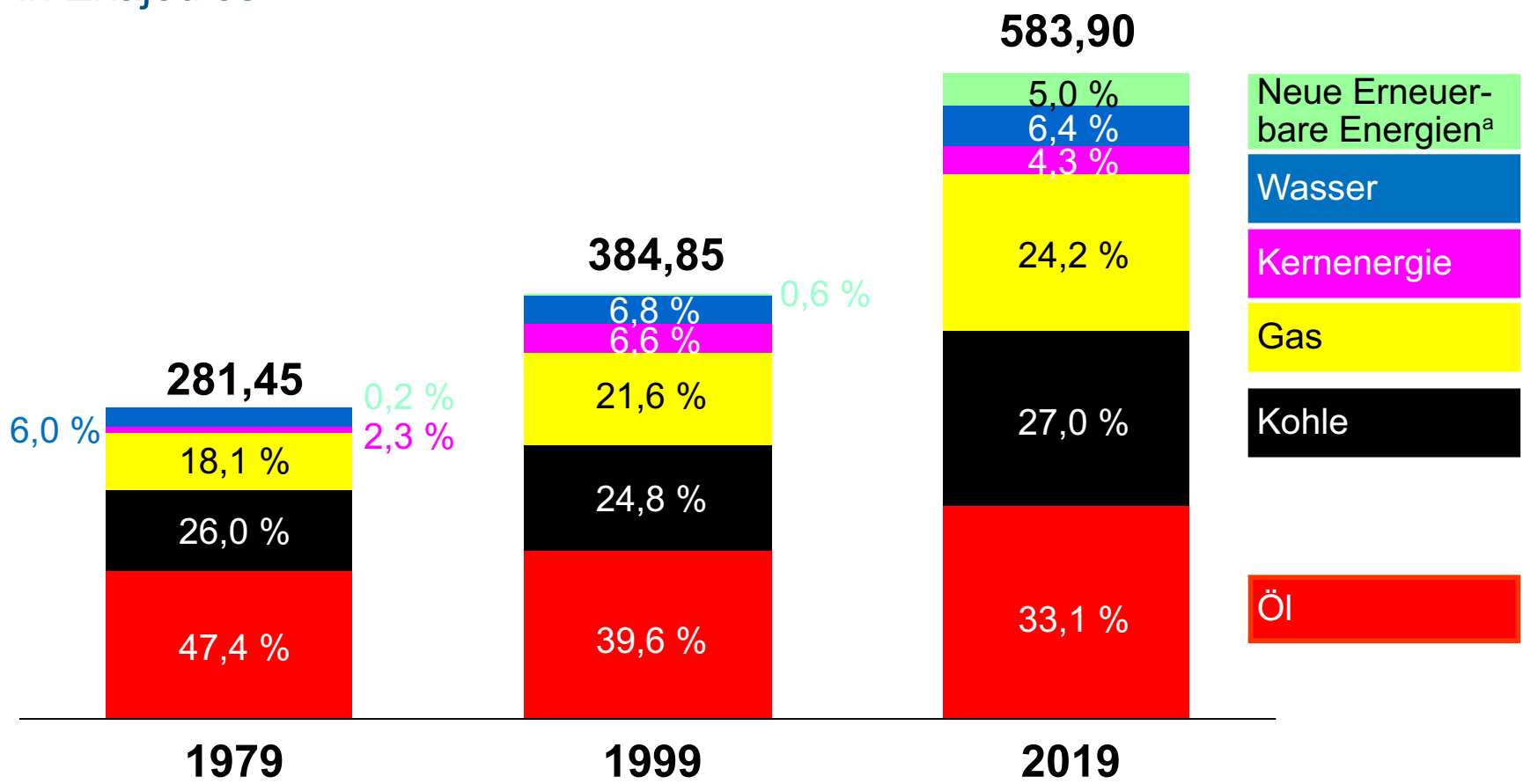

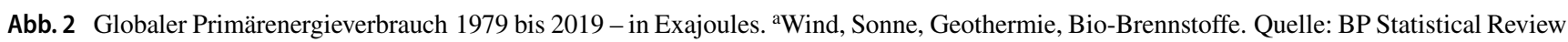
of World Energy June 2020 


\section{Primärenergieverbrauch weltweit - Synopse der exploratorischen Szenarien von Equinor und WEC bis 2050 in Milliarden Tonnen Öläquivalent (bn toe)}

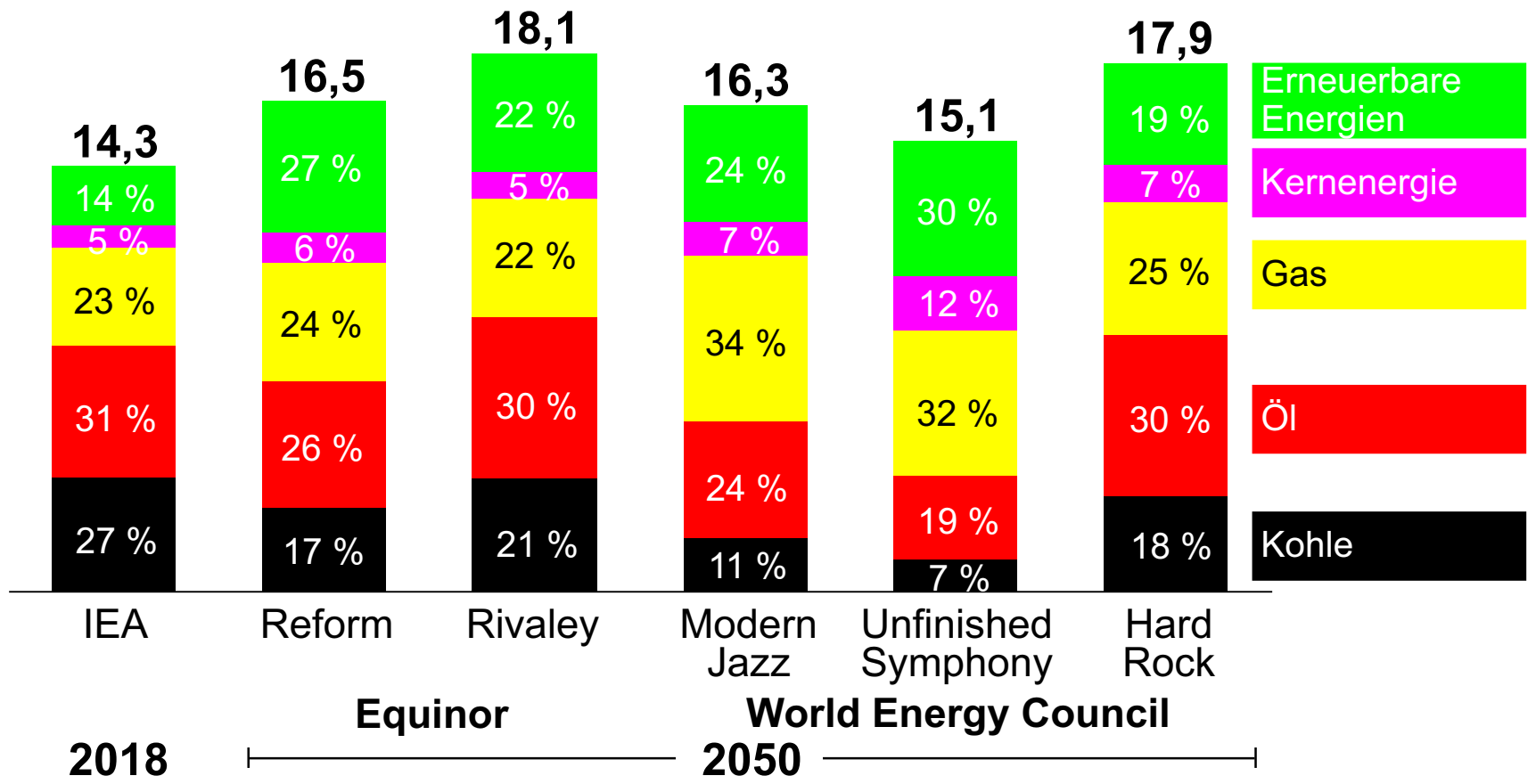

Abb. 3 Primärenergieverbrauch weltweit - Synopse der exploratorischen Szenarien von Equinor und WEC bis 2050 - in Milliarden Tonnen Öläquivalent (bn toe). Quelle: IEA, World Energy Outlook (2019); Equinor, Energy Perspectives (2019); World Energy Council, World Energy Scenarios $(2019 b)$

raum bis zum Jahr 2050 stützt. Kosten- und „Performance“Verbesserungen werden auf Basis historischer Trends berücksichtigt. Mögliche Veränderungen, etwa künftige technologische Durchbrüche oder politische Neuausrichtungen, die zu Anpassungen von Gesetzen, Verordnungen und staatlichen Zielen führen, sind nicht in Rechnung gestellt. Zur Quantifizierung nutzt die EIA das World Energy Projection System Plus (WEPS+), ein integriertes ökonomisches Modell, das langfristige Beziehungen zwischen Energieversorgung, Nachfrage und Preisen in sechszehn Weltregionen unter Berücksichtigung der genannten Annahmen erfasst.

\subsection{Methodik und Modellierung bei den Ansätzen von WEC und IEA}

Ebenfalls einem einzigen Cluster, in diesem Fall der Kategorie exploratorische Szenarien, lassen sich die drei vom World Energy Council (WEC) entwickelten Szenarien zuordnen. Der WEC hatte - unterstützt durch Accenture Strategy Energy - zum World Energy Congress im September 2019 in Abu Dhabi die Ergebnisse von drei plausiblen alternativen Pfaden für eine Transformation der weltweiten
Energieversorgung bis 2060 vorgelegt. Bei der Benennung der berücksichtigten drei Szenarien hat man sich zur Veranschaulichung mit Modern Jazz, Unfinished Symphony und Hard Rock verschiedener Musik-Richtungen bedient. Diese drei Szenarien können wie folgt charakterisiert werden:

- Modern Jazz ist als marktgetriebener Ansatz zu verstehen, gekennzeichnet durch eine starke Umsetzung technologischer Innovationen. Unternehmen bestimmen mittels individuell getroffener Entscheidungen die Dynamik. Das Szenario führt infolge der Realisierung der wirtschaftlichsten Lösungen $\mathrm{zu}$ dem höchsten Wirtschaftswachstum und der stärksten Verbesserung des Zugangs aller Menschen zu bezahlbarer Energie.

- Unfinished Symphony folgt einem durch Regierungen getriebenen Ansatz, gekennzeichnet durch umfassende politische Steuerung zur Umgestaltung der Energieversorgung. Weitere Kennzeichen sind eine ausgeprägte globale Kooperation vor allem beim Schutz des Klimas. Auch aufgrund der angesetzten höchsten Bepreisung von $\mathrm{CO}_{2}$ kommt es im Vergleich der Szenarien zu dem günstigsten Verlauf der Treibhausgas-Emissionen. 


\section{Synopse der Projektionen von DNV-GL, McKinsey, ExxonMobil, BP, IEA und EIA zum globalen Primärenergie- verbrauch bis 2040}

in Milliarden Tonnen Öläquivalent (bn toe)

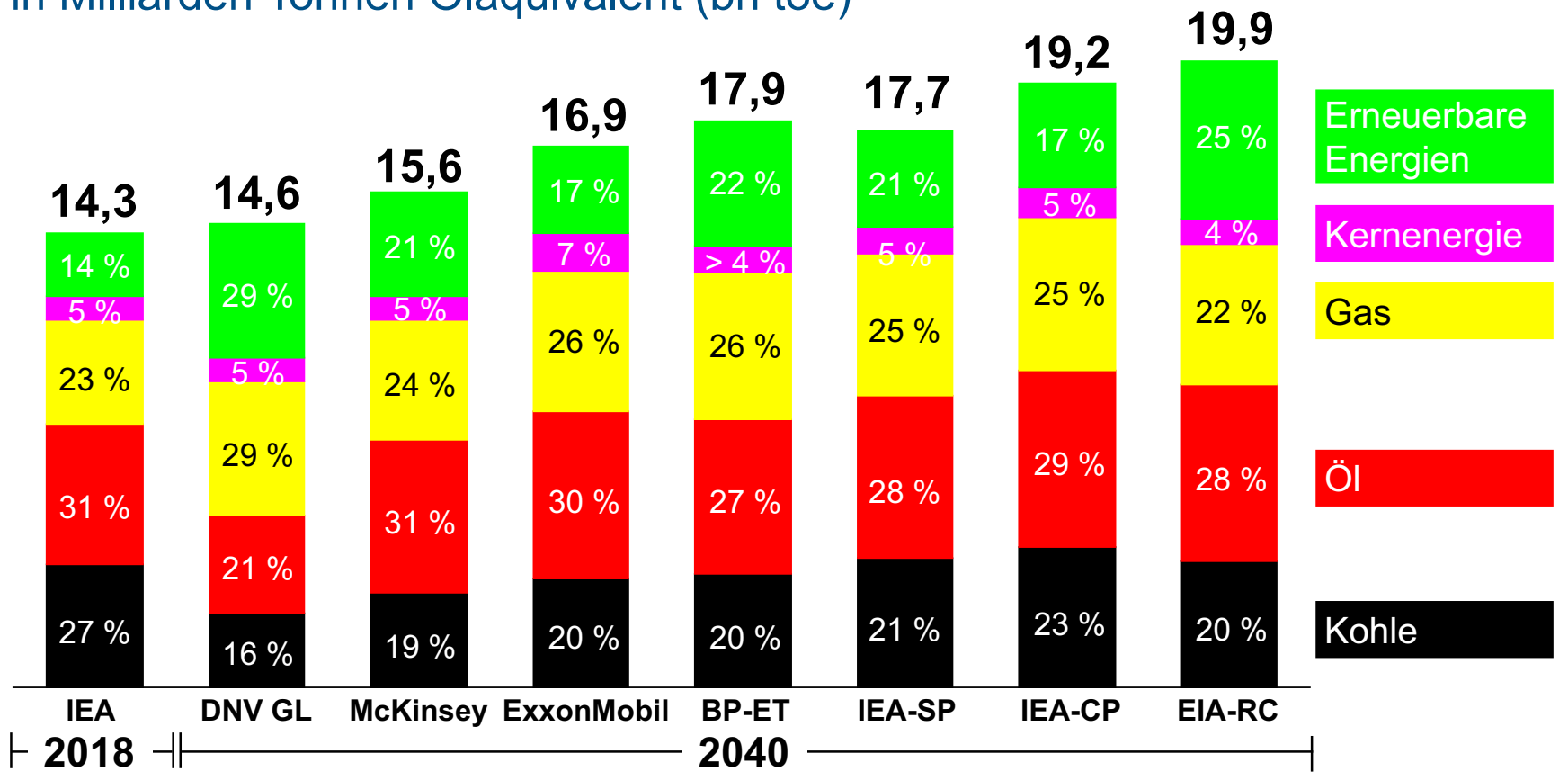

Abb. 4 Synopse der Projektionen von DNV-GL, McKinsey, ExxonMobil, BP, IEA und EIA zum globalen Primärenergie-verbrauch bis 2040 in Milliarden Tonnen Öläquivalent (bn toe). Quelle: EIA, International Energy Outlook (2019); IEA, World Energy Outlook (2019); BP Energy Outlook - (2019) Edition; ExxonMobil (2019) Outlook for Energy: A Perspective to 2040; DNV GL Energy Transition Outlook (2019)

- Hard Rock ist durch ein Patchwork aus Markt und Staat sowie durch eine fragmentierte Welt mit geringer internationaler Kooperation gekennzeichnet. Die Verfolgung nationaler Interessen steht im Vordergrund. Dem Gesichtspunkt der Sicherheit der Versorgung unter möglichst weitgehender Nutzung heimischer Energiequellen wird der größte Stellenwert beigemessen. Den Nachhaltigkeitszielen wird dieses Szenario am wenigsten gerecht.

Im Vordergrund dieser exploratorischen Szenarien stehen qualitative Storylines, die plausible alternative Zukunftspfade beschreiben und mittels einer durch das Paul Scherrer Institut vorgenommenen Modellierung quantitativ unterstützt sind. Die Szenarien wurden von einer mit Experten aus der gesamten Welt besetzten Study Group, geleitet von der Londoner Zentrale des World Energy Council, entwickelt. Die Quantifizierung der Szenarien erfolgte durch das Paul Scherrer Institut unter Einsatz des Global Multi-regional MARKAL (GMM)-Modells. Das Modell basiert auf InputParametern, welche die jeweiligen Storylines der verschiedenen Szenarien widerspiegeln, und es bestimmt die kosten- günstigsten Konfigurationen des globalen Energiesystems aus der Perspektive eines Planers mit perfekter Voraussicht. Das GMM-Modell repräsentiert das globale Energiesystem, disaggregiert in 17 Weltregionen einschließlich der spezifischen Charakteristika von Energie-Angebot und -Nachfrage in den jeweiligen Regionen sowie der dadurch bedingten Treibhausgas-Emissionen. Die Iteration zwischen der Entwicklung der Narrative und ihrer Quantifizierung legte den Grundstein für das skizzierte belastbare Set von Szenarien.

Das GMM-Modell bildet im Detail das Energiesystem der verschiedenen Weltregionen von der Quelle der verschiedenen Ressourcen bis zum Endverbrauch an Energie ab. Es schließt mehr als 400 Energie-Umwandlungstechnologien mit ihren technischen, ökonomischen und umweltrelevanten Merkmalen ein. Neben konventionellen Technologien bezieht das Modell $\mathrm{CO}_{2}$-freie Technologien sowie auch Optionen ein, mit denen negative $\mathrm{CO}_{2}$-Emissionen erzielt werden können, wie mittels des Einsatzes von Biomasse zur Stromerzeugung unter Abscheidung und Speicherung des $\mathrm{CO}_{2}$. Das Modell optimiert die gesamten diskontierten Energie-Systemkosten über den kompletten Modell-Horizont. Nicht durch Kosten belegbare Parameter sowie Ver- 


\section{Entwicklung des globalen Primärenergieverbrauchs in Zielszenarien bis 2050 in Mtoe}

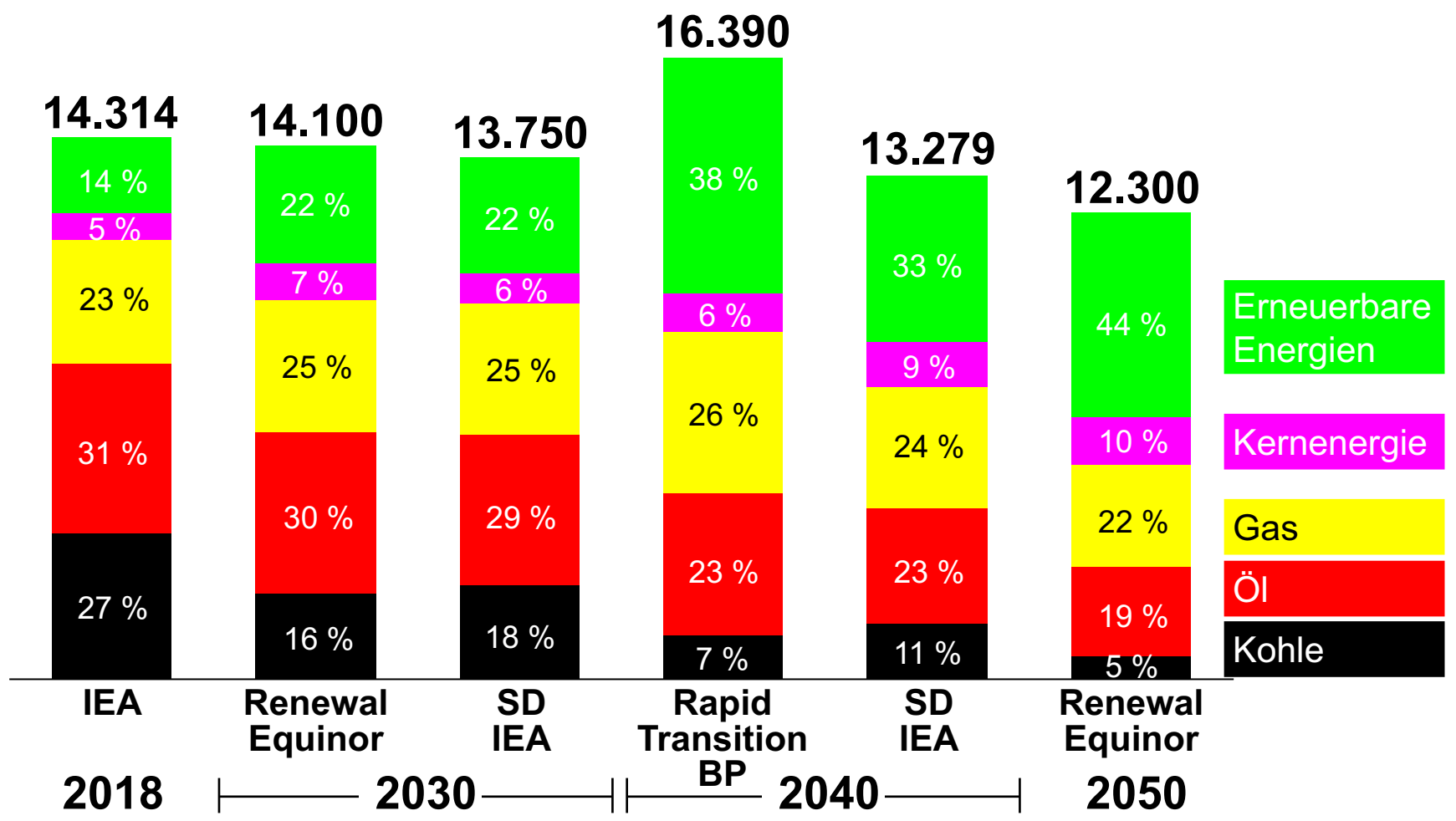

Abb. 5 Entwicklung des globalen Primärenergieverbrauchs in Zielszenarien bis 2050 - in Mtoe. Quelle: IEA, World Energy Outlook (2019); BP Energy Outlook (2019); Equinor, Energy Perspectives (2019)

haltensannahmen werden als Nebenbedingungen in der dem Modell zugrunde liegenden Zielfunktion berücksichtigt.

In allen drei Szenarien wird von einer Verlangsamung des Anstiegs der Weltbevölkerung im Vergleich zu Trends der Vergangenheit ausgegangen. So wird der Stand der Weltbevölkerung im Jahr 2060 auf 10,2 Mrd. angesetzt; dies entspricht einem Zuwachs um knapp ein Drittel im Vergleich zum gegenwärtigen Stand. Während die Bevölkerungszahl in den drei Szenarien in gleicher Höhe in das Modell eingeht, stellen sich die Annahmen zum Wirtschaftswachstum unterschiedlich dar. So wird von jahresdurchschnittlichen Wachstumsraten der weltweiten Wirtschaftsleistung von 3,3\% in Modern Jazz, 2,7\% in Unfinished Symphony und 2,2\% in Hard Rock ausgegangen. Unterschiedliche Ansätze sind auch hinsichtlich der Preisannahmen für $\mathrm{CO}_{2}$ getroffen. So steigen die $\mathrm{CO}_{2}$-Preise in Unfinished Symphony am stärksten an. Die $\mathrm{CO}_{2}$-Preise sind in den bevorstehenden Jahrzehnten zunächst noch unterschiedlich hoch nach Weltregionen angesetzt. Allerdings wird ab Mitte des Jahrhunderts in diesem Szenario von einem weltweit einheitlichen $\mathrm{CO}_{2}$-Preis ausgegangen, der sich 2050 auf 90US\$ (2010) und 2060 auf 110 US\$ (2010) pro Tonne beläuft. Im Unterschied dazu bleiben die $\mathrm{CO}_{2}-$ Preise in den anderen Szenarien deutlich niedriger und selbst im Jahr 2060 noch regional unterschiedlich hoch. Die für das Jahr 2060 berücksichtigten Spannen reichen von 19 bis 45 US\$ (2010) pro Tonne im Szenario Hard Rock bis 60 bis 90US\$ (2010) pro Tonne im Szenario Modern Jazz.

Ebenfalls drei Szenarien hat die Internationale EnergieAgentur (IEA) veröffentlicht - zuletzt im November 2019. Allerdings haben die Szenarien der IEA einen anderen Charakter. Zwei der berücksichtigten Pfade, nämlich Current Policies und Stated Policies, sind der Kategorie Projektionen zuzuordnen. Bei dem dritten Pfad, Sustainable Development, handelt es sich um ein normatives Szenario. Wie die IEA ausweist, ist keines der drei Szenarien als Prognose zu verstehen. Vielmehr handelt es sich um - unter den getroffenen Annahmen - mögliche konsistente Zukunftsbilder. 


\section{Globale Nachfrage nach fossilen Energieträgern gemäß den WEC-Szenarien}

in Mrd. t Öläquivalent

\section{Kohle-Nachfrage}

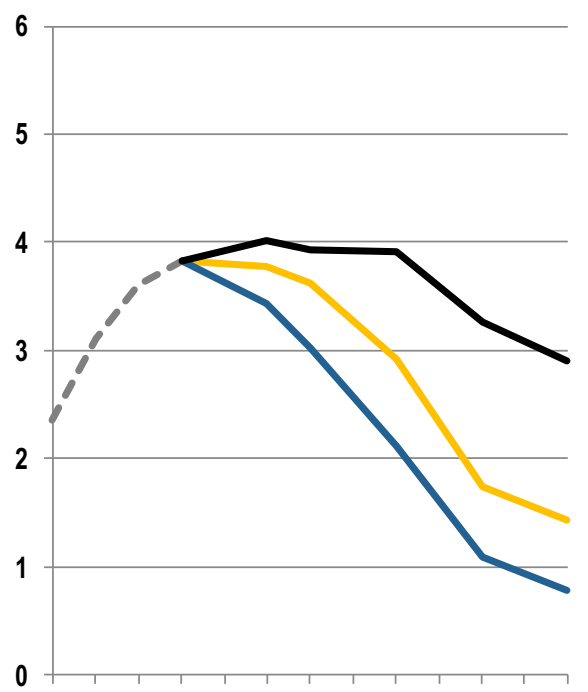

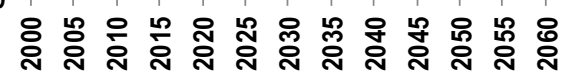

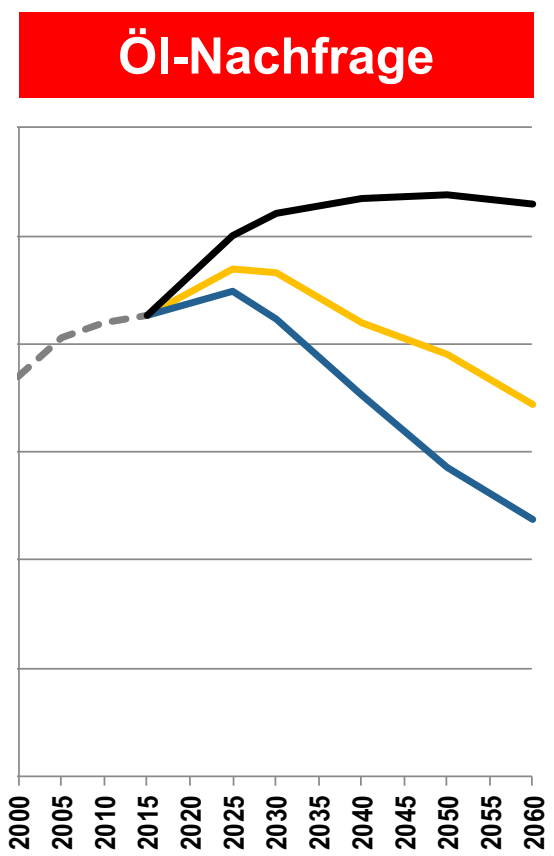

Öl-Nachfrage
Erdgas-Nachfrage

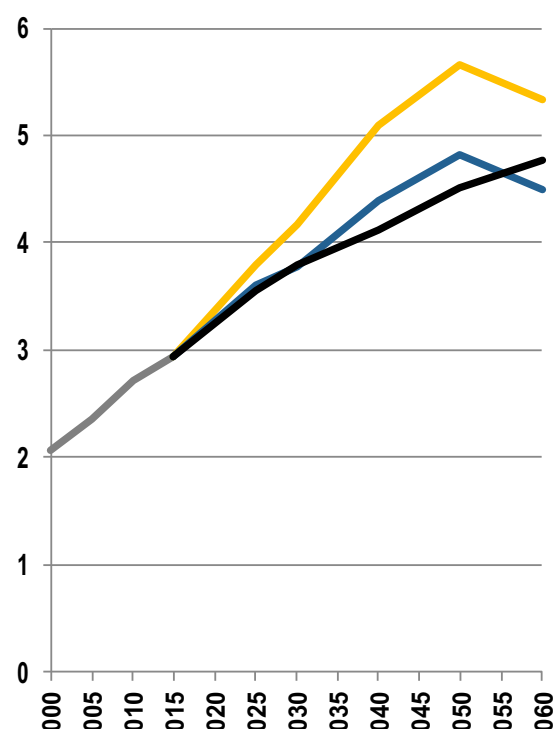

\section{- - Historie}

Modern Jazz

Unfinished Symphony

Hard Rock

Abb. 6 Globale Nachfrage nach fossilen Energieträgern gemäß den WEC-Szenarien - in Mrd.t Öläquivalent. Quelle: World Energy Council, Paul Scherrer Institute, Accenture Strategy: World Energy Scenarios (2019b), September 2019

- Das Szenario Current Policies (CP) geht von unveränderten energie- und klimapolitischen Rahmenbedingungen aus, berücksichtigt also nur die bis Mitte 2019 rechtlich verbindlich in Kraft gesetzten Regelungen.

- Das Szenario Stated Policies (SP) zeigt auf, wie sich das Energiesystem bei Zugrundelegung der aktuellen Politik und der bis Mitte 2019 angekündigten (stated) Pläne entwickeln könnte.

- Das Szenario Sustainable Development (SD) beschreibt einen integrierten Ansatz zur Verwirklichung der energiebezogenen Aspekte der UN-Ziele für nachhaltige Entwicklung, d.h. entschlossene Klimaschutzmaßnahmen im Einklang mit dem Pariser Klima-Abkommen, universeller Zugang zu zeitgemäßer Energie bis 2030 und drastische Reduzierung der Luftverschmutzung. Dies sind die drei Bereiche, in denen die Entwicklung in den anderen beiden Szenarien hinter den Erfordernissen zurückbleibt.

Stated Policies wird von der IEA als das zentrale Szenario klassifiziert. Diese Wahl macht, wie in der SzenarioTechnik üblich, keine Aussage über die Wahrscheinlichkeit des Eintretens oder über die Wünschbarkeit dieses oder eines der anderen beiden Szenarien.

Der Modellierung unterliegt ein Ansatz, der kostenminimierende Lösungen unter Berücksichtigung der in den drei Szenarien jeweils unterschiedlich definierten staatlichen Rahmenbedingungen ausweist. Dabei ist durch die Formulierung von Nebenbedingungen berücksichtigt, soweit bestimmte Technologiepfade durch administrative Maßnahmen - losgelöst von den Kostenverhältnissen begünstigt bzw. in ihrer Umsetzung begrenzt oder sogar ausgeschlossen werden. Beispielhalft können in diesem Zusammenhang der gesetzlich festgeschriebene Ausstieg aus der Kernenergie und aus der Kohle in Deutschland und der dort aufgrund gesetzlicher Regelungen praktisch ausgeschlossene Technologiepfad der Abscheidung und Speicherung von $\mathrm{CO}_{2}$ in der Verstromung fossiler Energieträger genannt werden.

Entscheidende Inputparameter bei der Modellierung der IEA-Szenarien sind die getroffenen Annahmen zur Entwicklung der Weltbevölkerung, zum Wirtschaftswachstum, zu den Weltmarktpreisen für Energie und zu den $\mathrm{CO}_{2}$-Preisen. Der Zeithorizont reicht bis 2040. Bis zu diesem Jahr 


\section{Weltweites Wachstum des Primärenergieverbrauchs nach Energieträgern (im Stated Policies Scenario)}
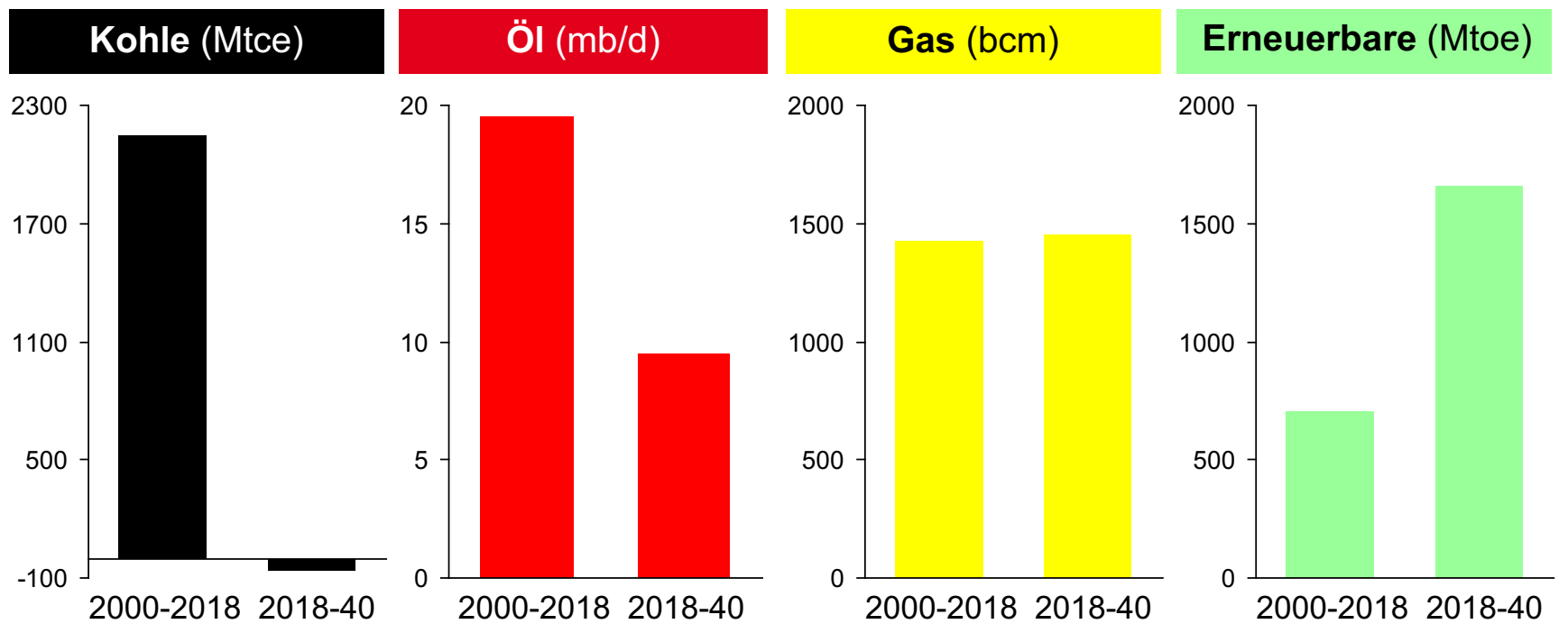

Abb. 7 Weltweites Wachstum des Primärenergieverbrauchs nach Energieträgern (im Stated Policies Scenario). Bei Erdgas Fortsetzung des Wachstumstrends der vergangenen Jahre; bei Öl stark verlangsamtes Wachstum; bei Kohle praktisch Stagnation. Erneuerbare Energien mit deutlich beschleunigter Wachstumsdynamik. Quelle: IEA, World Energy Outlook (2019)

geht die IEA von einem Anstieg der Weltbevölkerung auf 9,2 Mrd. Menschen aus. Es wird ein durchschnittliches reales Wirtschaftswachstum von 3,4\% pro Jahr im Zeitraum 2018 bis 2040 unterstellt. Die Weltmarktpreise für Energie sind - je nach Szenario - unterschiedlich hoch angesetzt. Sie sind tendenziell am höchsten im Szenario Current Policies und am niedrigsten im Szenario Sustainable Development. Dies erklärt sich durch die zwischen diesen Szenarien stark differierende Nachfrage nach fossilen Energieträgern. Die $\mathrm{CO}_{2}$-Preisannahmen unterscheiden sich sehr stark - nicht nur abhängig vom jeweiligen Szenario, sondern auch nach Staaten und Weltregionen. Die Spannweite reicht 2040 von 5 bis 39 US\$ (2018) pro Tonne in Current Policies, 20 bis 39 US\$ (2018) pro Tonne in Stated Policies und 125 bis 140US\$ (2018) pro Tonne in Sustainable Development. Die 125 US\$ (2018) sind für ausgewählte Entwicklungsländer und die 140 US\$ (2018) für wirtschaftlich fortgeschrittene Volkswirtschaften angenommen.

\subsection{Szenarien von Shell, BP und Equinor}

Der Energiekonzern Shell hatte im Jahr 2013 mit Mountains und Oceans zwei exploratorische Szenarien zur Entwicklung des globalen Energiesystems bis 2100 veröffentlicht. Diese bereits viele Jahre zurückliegende und deshalb nicht berücksichtigte Studie New Lens Scenarios war 2018 um das normative Szenario Sky ergänzt worden. Das Sze- nario Sky - Meeting the Goals of the Paris Agreement zeigt einen möglichen Pfad für eine Dekarbonisierung der globalen Wirtschaft mit Netto-Treibhausgas-Emissionen von Null aus der Nutzung von Energie bis zum Jahr 2070 auf. Bei der Erstellung des Szenarios Sky hat sich Shell auf das Integrated Global System Modelling (IGSM)-Rahmenwerk des Massachusetts Institute of Technology (MIT) Joint Program on the Science and Policy of Global Change gestützt. Damit war das Ziel verfolgt worden, den Kontrast zu den Szenarien Mountain und Oceans zu veranschaulichen (Shell International B.V. 2018).

Der Energiekonzern BP hatte im Februar 2019 den Energy Outlook to 2040 veröffentlicht. Dieser Outlook umfasst verschiedene Szenarien, bezeichnet mit Begriffspaaren wie Evolving Transition, More Energy, Less Globalization, Greater Reform und Rapid Transition. Im Vordergrund steht Evolving Transition (ET). Die verschiedenen Szenarien haben einige gemeinsame Merkmale; dazu gehören unter anderem die Erwartung eines fortgesetzten globalen Wirtschaftswachstums und des Übergangs zu einem Energiemix mit rückläufigen $\mathrm{CO}_{2}$-Emissionen. Die Szenarien unterscheiden sich aber bezüglich der Annahmen zur politischen Rahmensetzung, zur Umsetzung von Technologien und zu Verhaltensänderungen. Im Szenario Evolving Transition, das der Kategorie Projektionen zuzuordnen ist, wird davon ausgegangen, dass sich die Politiken der Regierungen, technologische Innovationen sowie Präferenzstrukturen der 


\section{9 bis 2040 erwarteter weltweiter Zubau von Stromerzeugungs-Kapazität von $8.473 \mathrm{GW}$ im Stated Policies Scenario}

(davon knapp ein Drittel Ersatz bestehender Anlagen) entspricht ...

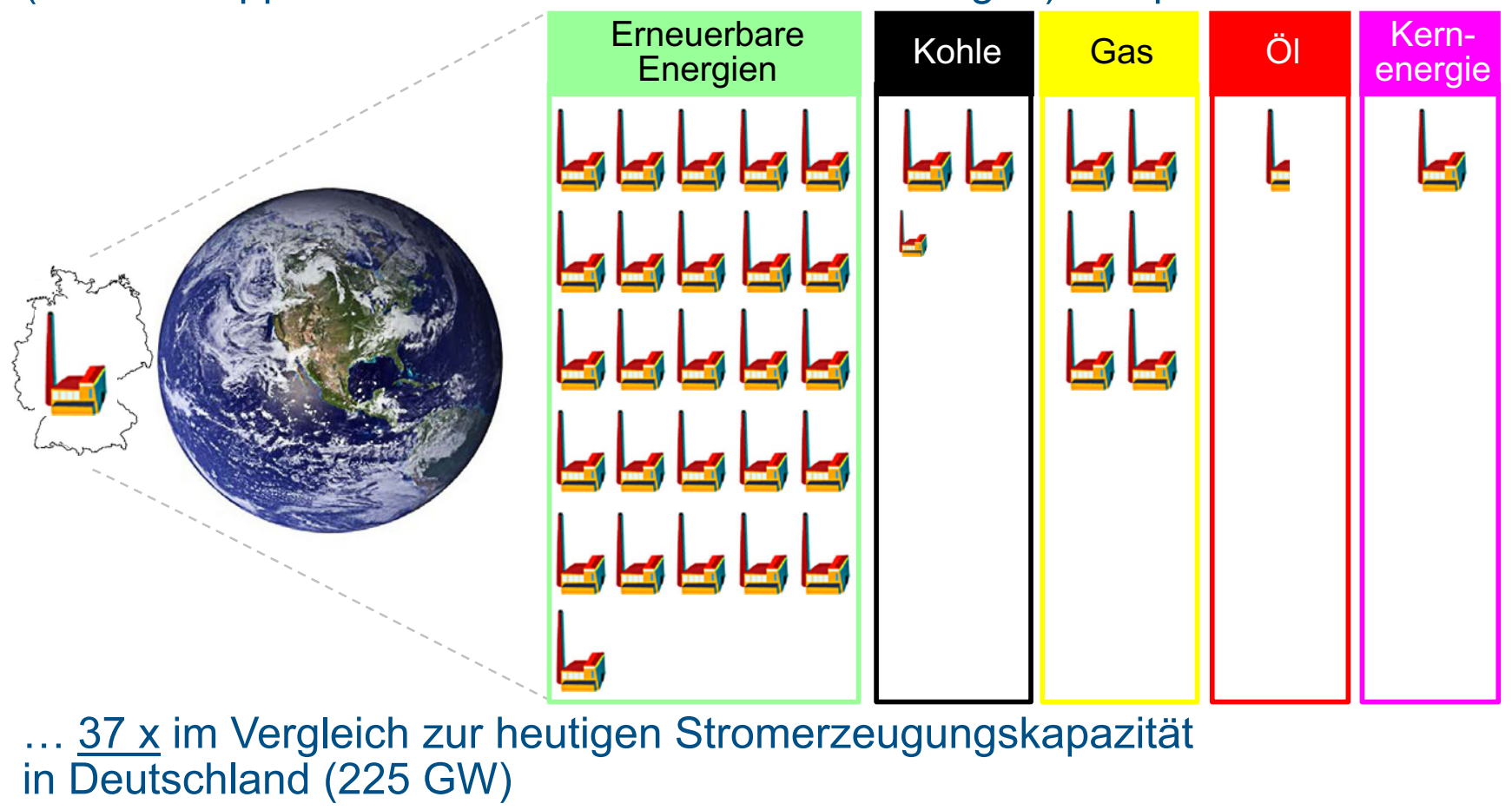

Abb. 82019 bis 2040 erwarteter weltweiter Zubau von Stromerzeugungs-Kapazität von 8473GW im Stated Policies Scenario (davon knapp ein Drittel Ersatz bestehender Anlagen) entspricht 37× im Vergleich zur heutigen Stromerzeugungskapazität in Deutschland (225 GW). Quelle: International Energy Agency, World Energy Outlook (2019, S. 676)

Gesellschaft in gleicher Geschwindigkeit entwickeln, wie dies für die jüngste Vergangenheit zu beobachten war. Das bedeutet aber nicht, dass die Eintrittswahrscheinlichkeit dieses Szenarios als höher im Vergleich zu den alternativ entwickelten Zukunftspfaden eingestuft wird. In einem der alternativen Szenarien, dem Rapid Transition (RT), wird ermittelt, was passieren müsste, um dem 2-Grad-Ziel gerecht zu werden.

Der Konzern Equinor, mehrheitlich in norwegischem Staatsbesitz, nutzt Szenarien, um zu beschreiben, wie Politik, Technologie und Marktbedingungen Entwicklungen in verschiedene Richtung auslösen können, in erwünschte und in unerwünschte. Drei Szenarien wurden ausgewählt, um mögliche globale Energiemarkt-Trends bis zum Jahr $2050 \mathrm{zu}$ analysieren, benannt mit Reform, Renewal und Rivalry. Reform, als das zentrale Szenario bezeichnet, charakterisiert eine Zukunft, die von technologischen Innovationen und durch Marktkräfte getriebene Entwicklungen, unterstützt von graduell verstärkten energie- und klimapolitischen Maßnahmen, bestimmt wird. Rivalry beschreibt eine Zukunft, in der sich die Transformation der Ener- gieversorgung verlangsamt, ausgelöst durch Mangel an Vertrauen, geopolitischer Stabilität und infolge der Wahl ineffizienter Lösungen zur Bewältigung der anstehenden Herausforderungen. Diese zwei exploratorischen Szenarien sind ergänzt um das normative Szenario Renewal. Darin wird aufgezeigt, wohin sich die Energiemärkte bewegen sollten, damit eine nachhaltige Zukunft erreicht werden kann. Renewal ist getrieben von einer schnellen und signifikanten Verstärkung der Energie- und Klimapolitik sowie globaler Kooperation und rasantem technologischen Wandel mit der Konsequenz, dass sich die TreibhausgasEmissionen auf einem Pfad bewegen, der mit dem Pariser Klima-Übereinkommen konsistent ist. Als analytische Basis für diesen Outlook wird die langfristige Forschung zu Fragen der Makroökonomie und der Energiemärkte genannt, die in enger Kooperation der Experten aus allen diesbezüglich relevanten Bereichen des Unternehmens praktiziert wurde. 


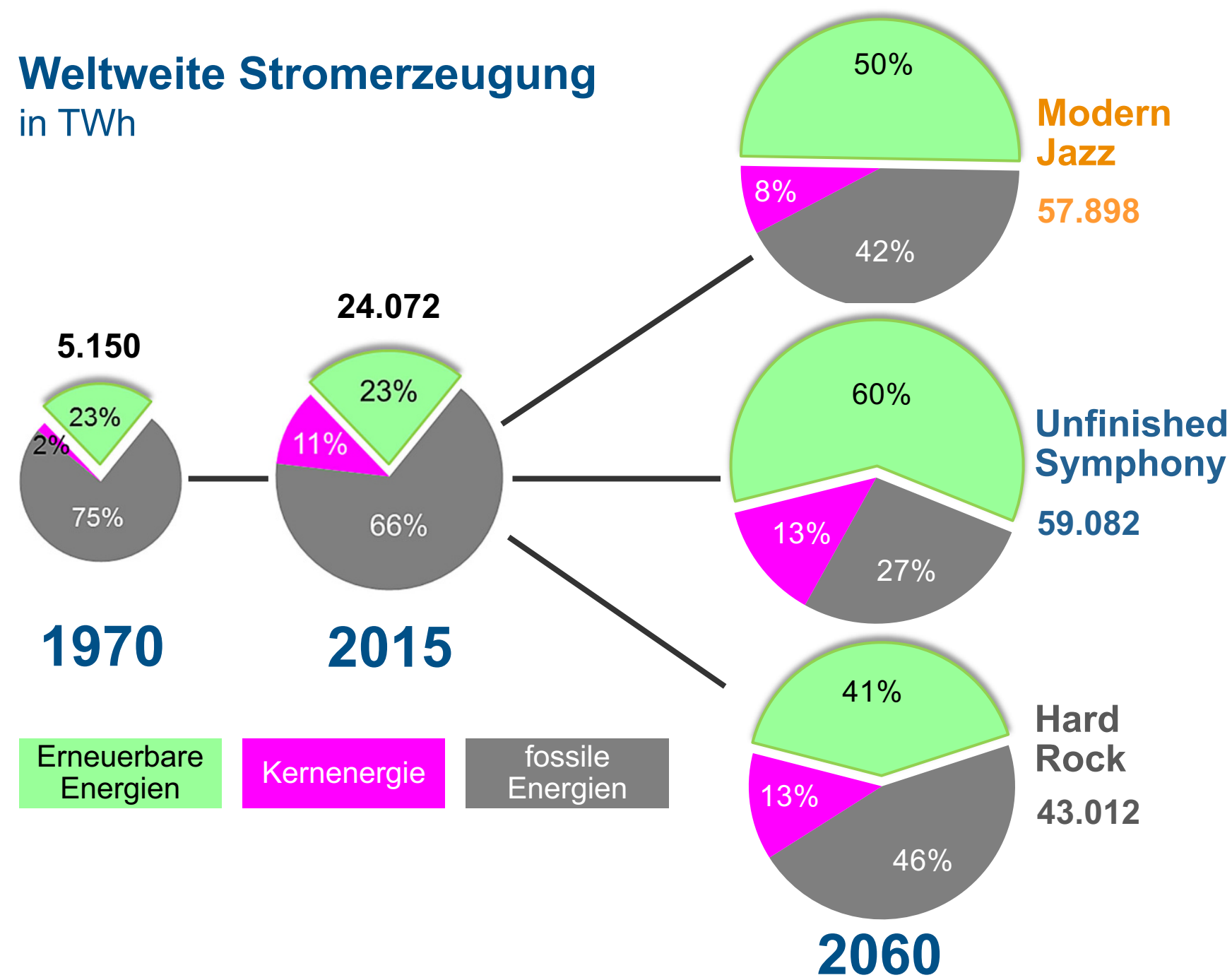

Abb. 9 Weltweite Stromerzeugung - in TWh. Quelle: H.-W. Schiffer auf Basis von: BP Statistical Review of World Energy und World Energy Council, Paul Scherrer Institute, Accenture Strategy: World Energy Scenarios (2019b), September 2019

\section{Zentrale Botschaften der berücksichtigten Studien}

Trotz aller Unterschiede zwischen den vorliegenden Studien lassen sich Gemeinsamkeiten in den zentralen Aussagen erkennen. Abweichungen in Bezug auf konkrete quantitativ unterlegte Ergebnisse erklären sich vielfach durch Unterschiede im Charakter der dargelegten Zukunftspfade sowie durch die getroffenen Grundannahmen.

\subsection{Zur Entwicklung des gesamten Primärenergieverbrauchs}

In den vergangenen vier Jahrzehnten hat sich der weltweite Primärenergieverbrauch mehr als verdoppelt (Abb. 2). Entscheidende Treiber waren der Anstieg der Weltbevölkerung von 4,4 Mrd. im Jahr 1979 um 75\% auf 7,7 Mrd. im Jahr
2019 sowie die in diesem Zeitraum verdreifachte globale Wirtschaftsleistung - gemessen in konstanten US\$, also in realen Größen.

Für die unmittelbar bevorstehenden Jahrzehnte ist zwar gemäß den meisten analysierten Studien noch eine Fortsetzung des Wachstums des Primärenergieverbrauchs zu erwarten, allerdings mit einer im Vergleich zur Vergangenheit deutlich geringeren Intensität. Die Entwicklung und der Einsatz neuer Technologien werden den Verbrauch im Vergleich zu historischen Trends deutlich dämpfen.

In den exploratorischen Szenarien und den Projektionen ergeben sich für den Zeitraum bis 2040 Zuwachsraten, die im Mittel halb so hoch sind wie die Steigerungsraten der vergangenen Jahrzehnte (Abb. 3 und 4). So wird beispielsweise im Stated Policy Scenario der IEA bis 2040 noch ein Anstieg des Primärenergieverbrauchs um 24\% ausgewiesen. Vergleichbare Größenordnungen nennen das Szenario 


\section{Globale Stromerzeugung aus erneuerbaren Energien}
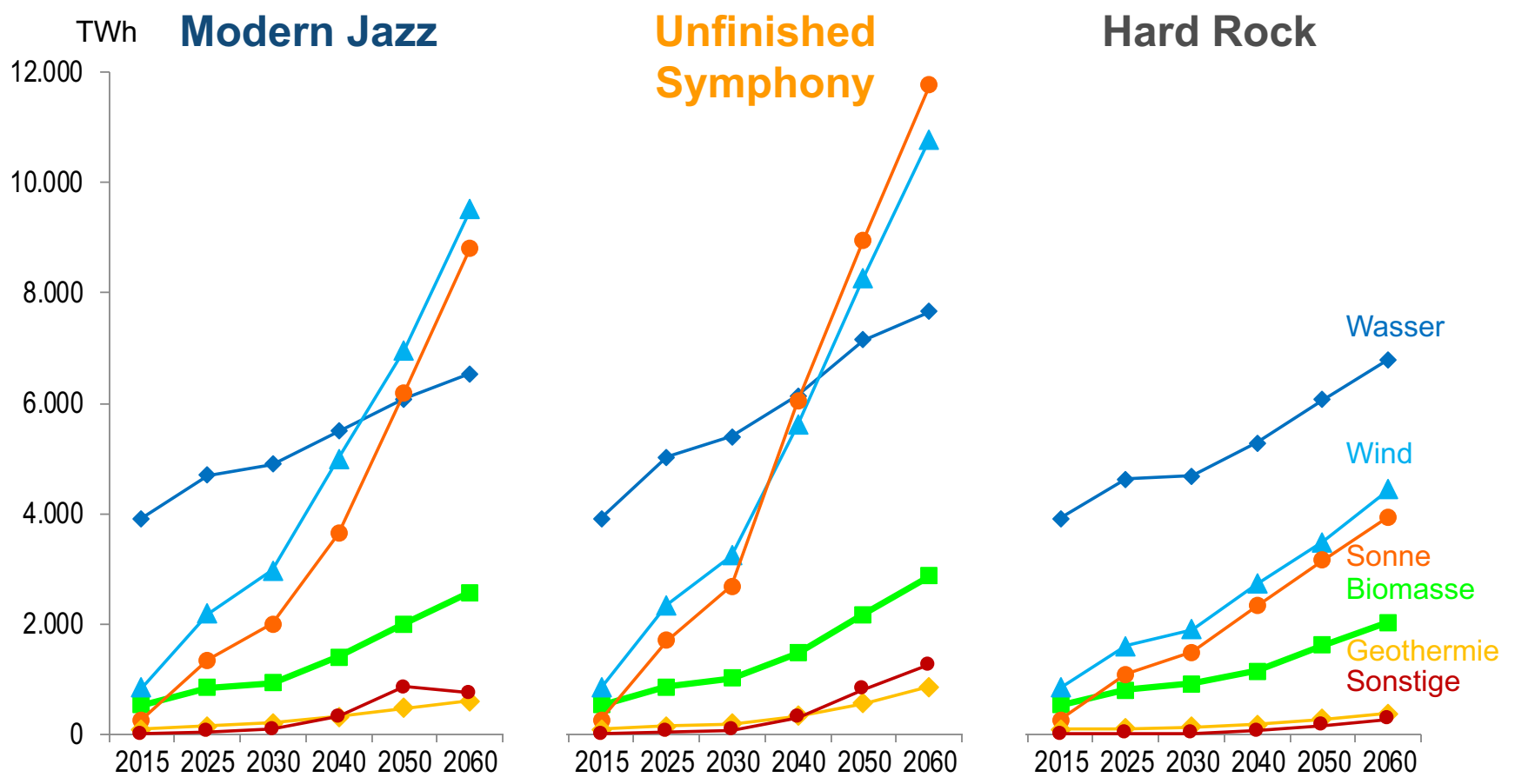

Abb. 10 Globale Stromerzeugung aus erneuerbaren Energien. Quelle: World Energy Council, Paul Scherrer Institute, Accenture Strategy: World Energy Scenarios (2019), September 2019

ET von BP, das Szenario Reform von Equinor und die Prognose von ExxonMobil. Die drei exploratorischen Szenarien des World Energy Council (WEC) decken sich mit dieser Tendenz. McKinsey beziffert den noch zu erwartenden Verbrauchszuwachs auf weniger als $10 \%$. Und DNV-GL kommt zu dem Ergebnis, dass der Höhepunkt des weltweiten Verbrauchs im Jahr 2030 erreicht wird.

Die quantitativen Ergebnisse der WEC-Studie, die bis 2060 reichen, kommen in allen Szenarien zu einer weiteren Abflachung der Verbrauchskurve nach 2040. In Unfinished Symphony steigt der Primärenergieverbrauch nach 2040 kaum noch an. In Modern Jazz wird Peak Demand im Jahr 2050 und in Hard Rock im Jahr 2060 erreicht.

In den normativen Szenarien ist sogar ein absoluter Rückgang im weltweiten Primärenergieverbrauch bereits innerhalb des Zeithorizonts bis 2040/2050 in Ansatz gebracht (Abb. 5).

Schlüsselbotschaft Nach einer verzeichneten Verdopplung des weltweiten Primärenergieverbrauchs in den letzten vier Jahrzehnten flacht sich die Nachfragekurve künftig deutlich ab.

\subsection{Wandel im Energiemix}

Die vergangenen Jahrzehnte waren durch eine Dominanz der fossilen Energieträger gekennzeichnet. Mehr als drei Viertel des Zuwachses im Primärenergieverbrauch, der zwischen 1979 und 2019 zu verzeichnen war, ist durch fossile Energieträger gedeckt worden. Alle fossilen Energieträger haben in absoluten Größen steigende Beiträge zur Deckung des Bedarfs geleistet. Überproportional stark hat Erdgas zugelegt. Der Anteil von Kohle war weitgehend stabil geblieben. Damit konnte Kohle seine Rolle als zweitwichtigster Energieträger hinter Öl und vor Erdgas bis 2019 behaupten. Im Zuge des verringerten Anteils von Öl hat sich der Beitrag fossiler Energieträger zur Deckung des Primärenergieverbrauchs von 91,5 auf 84,3\% im Jahr 2019 verringert.

Die Bedeutung der Kernenergie hatte sich bis 1999 kontinuierlich erhöht. Seitdem ist der Primärenergieverbrauch an Kernenergie - abgesehen von kleineren jährlichen Schwankungen - weitgehend stabil. Damit hat sich der Anteil der Kernenergie am Primärenergieverbrauch, der sich bis 1999 auf 6,6\% im Vergleich zum Stand des Jahres 1979 fast verdreifacht hatte, bis 2019 auf 4,3\% verringert.

Die erneuerbaren Energien haben, vor allem seit dem Jahr 2000, in relativen Größen zwar am stärksten zugelegt. Ihr Beitrag zur Deckung des globalen Primärenergiever- 


\section{Verschiedene Szenarien im Vergleich Globale Stromerzeugung 2018 bis 2040}

in TWh

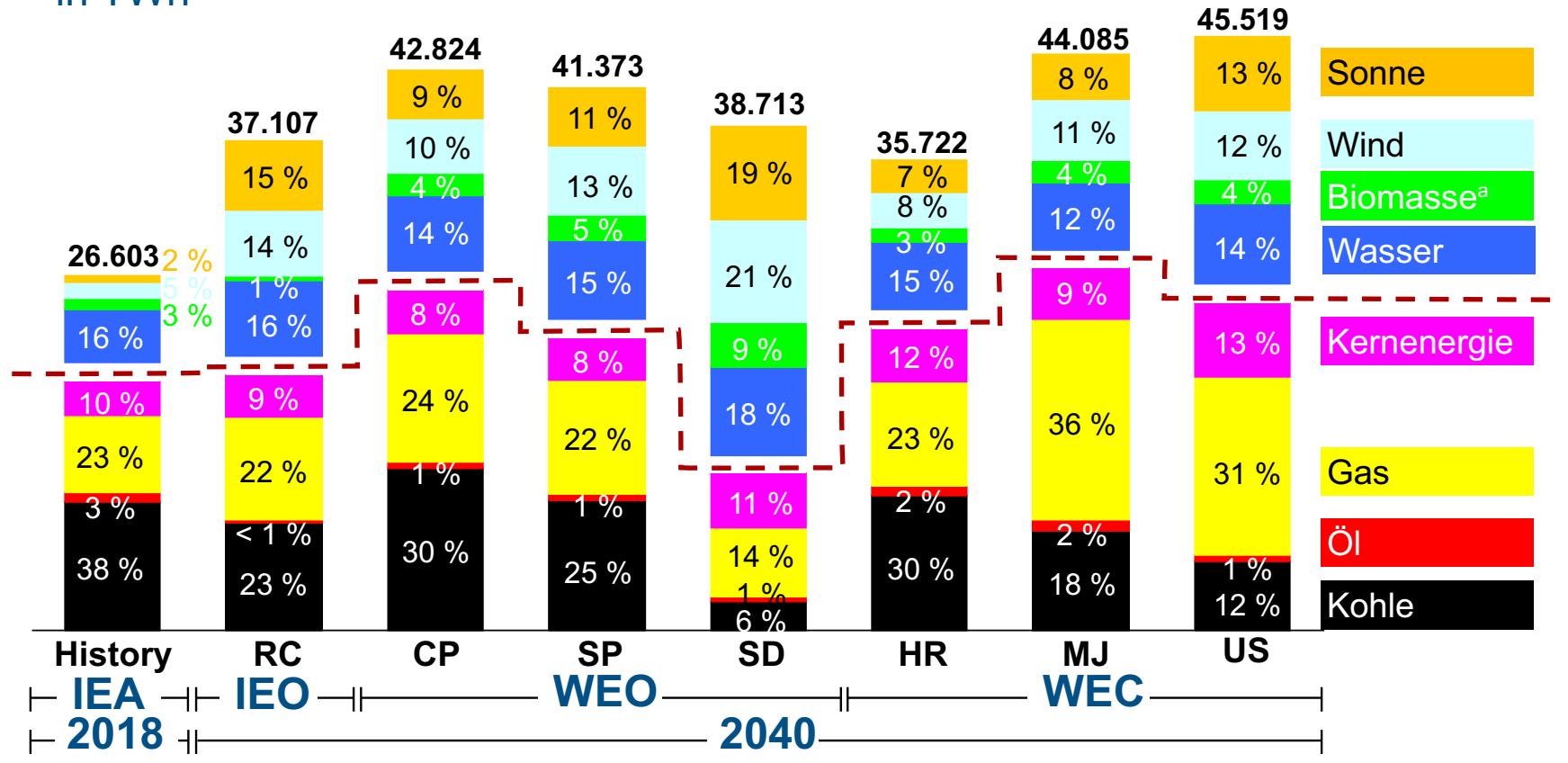

Abb. 11 Verschiedene Szenarien im Vergleich Globale Stromerzeugung 2018 bis 2040 - in TWh. ${ }^{a}$ Einschließlich Geothermie. Quelle: EIA, IEO (2019); IEA, WEO (2019); WEC, World Energy Scenarios (2019b)

brauchs war 2019 - gemäß BP Statistical Review of World Energy June 2020 - gleichwohl noch auf $11,4 \%$ begrenzt. Die IEA weist höhere Anteile aus, weil in deren Statistik auch die nicht-kommerzielle Biomasse einbezogen ist.

Die Zukunft wird deutlich anders eingeschätzt. Wichtigste Merkmale sind: Soweit noch ein Zuwachs im Primärenergieverbrauch zu erwarten ist, wird dieser überwiegend durch steigende Beiträge der erneuerbaren Energien gedeckt. Der Anteil fossiler Energien vermindert sich deutlich, bei DNV-GL von gegenwärtig noch mehr als 80 auf 66\% im Jahr 2040 und 56\% im Jahr 2050. Die gleiche Tendenz kommt in den Szenarien und Prognosen der anderen Institutionen zum Ausdruck, auch wenn darin zum Teil noch etwas höhere Anteilswerte für die fossilen Energien ausgewiesen werden als bei DNV-GL.

Innerhalb der Gruppe der fossilen Energien geht die Entwicklung am stärksten zulasten der Kohle, während Erdgas sich in den bevorstehenden Jahrzehnten noch weitgehend behaupten kann. So ist davon auszugehen, dass die Verbrauchskurve bei Kohle den Höhepunkt bereits überschritten hat und sich die seit 2013/2014 abzeichnende Abschwächung des globalen Kohleverbrauchs ab 2020 verschärft fortsetzen wird. Peak Demand für Öl wird zum Beispiel im Stated Policy Scenario der IEA um das Jahr 2030 und für Gas im Jahr 2040 erwartet. McKinsey rechnet mit Peak Demand für Öl im Jahr 2032 und für Gas im Jahr 2036.

Die Modellrechnungen des WEC weisen in den Szenarien Unfinished Symphony und Modern Jazz Peak Demand für Öl zwischen 2025 und 2030 aus. Für Hard Rock wird bis 2040 noch ein Anstieg gesehen, der danach in eine Plateauphase übergeht. Für Erdgas zeigen alle drei Szenarien des WEC noch bis 2050 einen steigenden Verbrauch (Abb. 6). Bis zum Jahr 2060 verringert sich der Anteil der fossilen Energien an der Deckung des Primärenergieverbrauchs auf $50 \%$ in Unfinished Symphony und auf $70 \%$ in Hard Rock. In Modern Jazz sind es 63\% - verglichen mit einem gegenwärtigen Anteil von rund $80 \%$.

In den Zielszenarien wird der Anteil fossiler Energien allerdings noch deutlich geringer eingeschätzt als in den Projektionen und den exploratorischen Szenarien. So kommen Equinor (Renewal), BP (Rapid Transition) und IEA (Sustainable Development) bereits für 2040 nur noch auf Anteile für die fossilen Energien zwischen 46 und 58\%.

Die sinkenden Anteile der fossilen Energien werden kompensiert durch steigende Beiträge der erneuerbaren Energien. Dies zeigen praktisch alle Projektionen und exploratorischen Szenarien. So kommen die Rechnungen der IEA im Stated Policy Scenario zu dem Ergebnis, dass sich der Primärenergieverbrauch der erneuerbaren Energien bis 


\section{Globale Stromerzeugung nach Energieträgern gemäß den Szenarien des WEC bis 2060 in TWh}

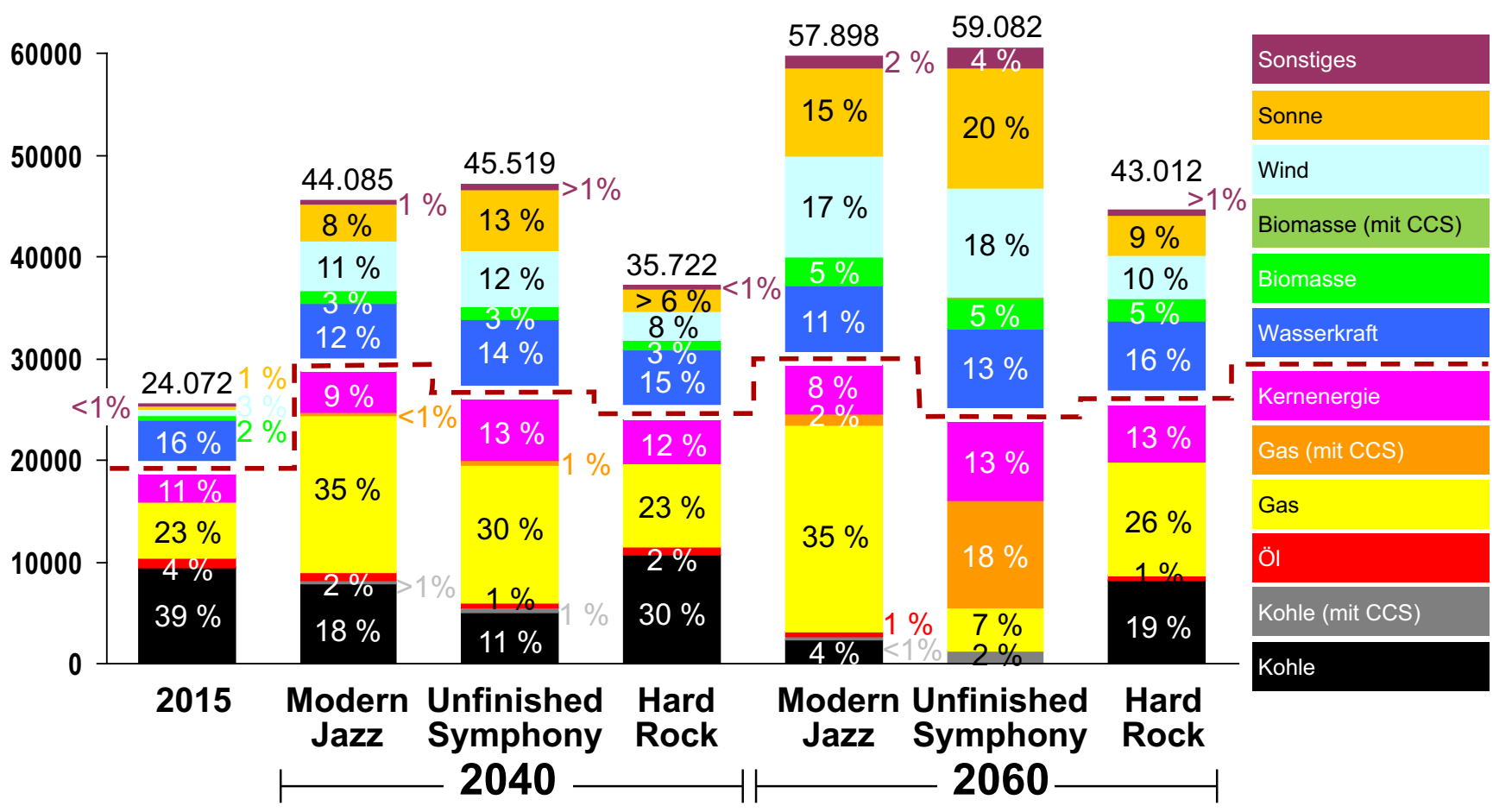

Abb. 12 Globale Stromerzeugung nach Energieträgern gemäß den Szenarien des WEC bis 2060 - in TWh. Quelle: World Energy Council, Paul Scherrer Institute, Accenture Strategy: World Energy Scenarios (2019b), September 2019

2040 im Vergleich zum Stand des Jahres mehr als verdoppelt, während der Verbrauch an Kohle im gleichen Zeitraum leicht zurückgeht und die zusätzliche Nachfrage nach Öl nur noch halb so hoch ausfallen wird wie im Zeitraum 2000 bis 2018. Bei Erdgas wird für 2018 bis 2040 ein Verbrauchszuwachs in gleicher Größenordnung wie im Zeitraum 2000 bis 2018 ermittelt (Abb. 7).

In den normativen Szenarien wird den erneuerbaren Energien eine noch darüber hinausgehende Rolle beigemessen. Sie werden bereits in den 2030er Jahren zur mengenmäßig wichtigsten Energiequelle und erreichen 2040 Anteilswerte am globalen Primärenergieverbrauch, die auf $33 \%$ (Sustainable Development Scenario der IEA) bis $44 \%$ (Renewal von Equinor) beziffert werden. Sie lassen damit Erdöl und Erdgas und vor allem die Kohle weit hinter sich. Der Kernenergie wird zwar in den normativen Szenarien eine wachsende Bedeutung eingeräumt. Als Game Changer wird diese $\mathrm{CO}_{2}$-freie Energiequelle jedoch nicht gesehen.

Schlüsselbotschaft Wir befinden uns am Wendepunkt vom fossilen Energiezeitalter zu einer durch erneuerbare Energien geprägten Welt.

\subsection{Entwicklung des globalen Stromverbrauchs und des Wandels im Erzeugungsmix}

Strom wird in allen Szenarien und Projektionen als Schlüsselenergie zur Dekarbonisierung der globalen Energieversorgung gesehen. Dies wird mit der Erwartung verknüpft, dass der Stromverbrauch in etwa doppelt so stark steigt wie der globale Primärenergieverbrauch.

Die eingeleitete Transformation der Energieversorgung zeigt sich in den Perspektiven für den künftigen Energiemix zur Stromerzeugung noch deutlicher als dies bei den Zahlen zum Primärenergieverbrauch zu erkennen ist. So wird der erwartete Stromverbrauchszuwachs ganz überwiegend durch den Ausbau von Anlagen auf Basis erneuerbarer Energien gedeckt. Laut IEA handelt es sich bei rund $70 \%$ der weltweit zwischen 2019 und 2040 neu installierten Anlagen zur Stromerzeugung um Leistung auf Basis von Sonne, Wind, Wasser, Biomasse, Geothermie und Meeresenergie (Abb. 8).

Besonders deutlich wird der Paradigmenwechsel durch Veranschaulichung der Ergebnisse der exploratorischen Szenarien des WEC für den Zeitraum 2015 bis 2060 


\section{Entwicklung des Energieverbrauchs - differenziert nach OECD- und Nicht-OECD-Staaten - gemäß 2019 Outlook for Energy von ExxonMobil}

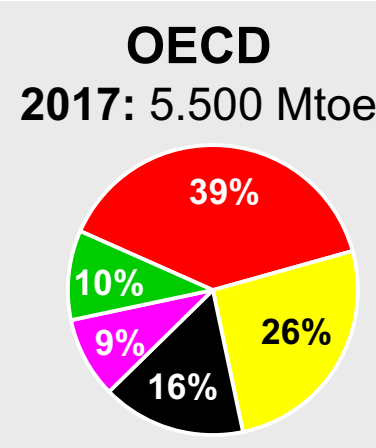

2040: 5.200 Mtoe

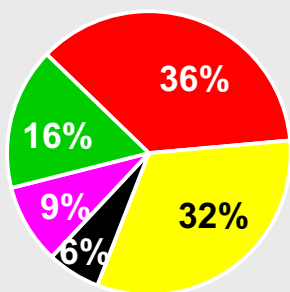

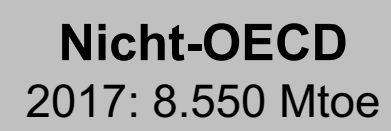

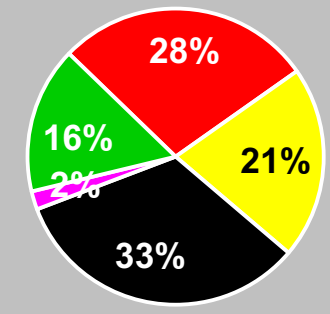

2040: 11.675 Mtoe

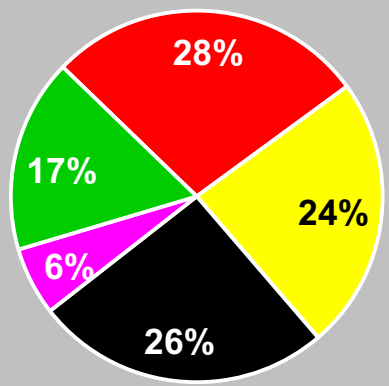

Öl

Gas

Kohle

Kernenergie

Erneuerbare Energien ${ }^{\mathrm{a}}$

Abb. 13 Entwicklung des Energieverbrauchs - differenziert nach OECD- und Nicht-OECD-Staaten - gemäß 2019 Outlook for Energy von ExxonMobil. ${ }^{a}$ einschließlich Biomasse/Abfall. Quelle: ExxonMobil (2019), Outlook for Energy: A Perspective to 2040

Tab. 1 Entwicklung der weltweiten gewichteten durchschnittlichen Investitionskosten, der Kapazitätsfaktoren und der Stromerzeugungskosten

\begin{tabular}{|c|c|c|c|c|c|c|}
\hline \multirow[t]{2}{*}{ Technologie } & \multicolumn{2}{|c|}{$\begin{array}{l}\text { Investitionskosten } \\
\text { in US } \$ / \mathrm{kW}\end{array}$} & \multicolumn{2}{|c|}{$\begin{array}{l}\text { Kapazitätsfaktor }{ }^{\mathrm{a}} \\
\text { in } \%\end{array}$} & \multicolumn{2}{|c|}{$\begin{array}{l}\text { Durchschnittliche Erzeugungskosten } \\
\text { in } 2019 \mathrm{US} \$ / \mathrm{kWh}\end{array}$} \\
\hline & 2010 & 2019 & 2010 & 2019 & 2010 & 2019 \\
\hline Utility Scale Solar PV & 4702 & 995 & 14,0 & 18,0 & 0,378 & 0,068 \\
\hline Onshore Wind & 1949 & 1473 & 27,1 & 35,6 & 0,086 & 0,053 \\
\hline Offshore Wind & 4650 & 3800 & 36,8 & 43,5 & 0,161 & 0,115 \\
\hline CSP & 8987 & 5774 & 30,0 & 45,2 & 0,346 & 0,182 \\
\hline Wasserkraft & 1254 & 1704 & 43,9 & 48,4 & 0,037 & 0,047 \\
\hline Bioenergie & 2588 & 2141 & 71,7 & 70,0 & 0,076 & 0,066 \\
\hline Geothermie & 2588 & 3916 & 87,0 & 79,4 & 0,049 & 0,073 \\
\hline
\end{tabular}

Quelle: IRENA (2020)

aBeim Kapazitätsfaktor handelt es sich um den aus der Zahl der Volllaststunden abgeleiteten Jahresnutzungsgrad (gerechnet bei $8760 \mathrm{~h}$ eines Jahres mit 365 Tagen)

${ }^{b}$ Die Levelized Cost of Electricity (LCOE) ergeben sich aus den abgezinsten Kapitalkosten, den fixen und variablen Betriebskosten, den Brennstoffkosten sowie der angestrebten Kapitalverzinsung über den Betriebszeitraum

im Vergleich zu den dieser Zeitspanne vorangehenden 45 Jahren. So war der Anteil erneuerbarer Energien an der Stromerzeugung 2015 mit $23 \%$ exakt genau so hoch wie im Jahr 1970. Im Unterschied dazu wird bis 2060 vom WEC ein Anstieg des Anteils erneuerbarer Energien an der globalen Stromerzeugung auf - je nach Szenario - Werte zwischen 41 und $60 \%$ geschätzt (Abb. 9).

Unter den erneuerbaren Energien erfolgt der stärkste Ausbau bei Solar- und Windanlagen. Diese Tendenz zeigen alle analysierten Szenarien und Projektionen. Bis 2060 wird 


\section{Zuwachs im Primärenergieverbrauch nach Weltregionen 2018 bis 2040 (im Stated Policy Scenario der IEA)}

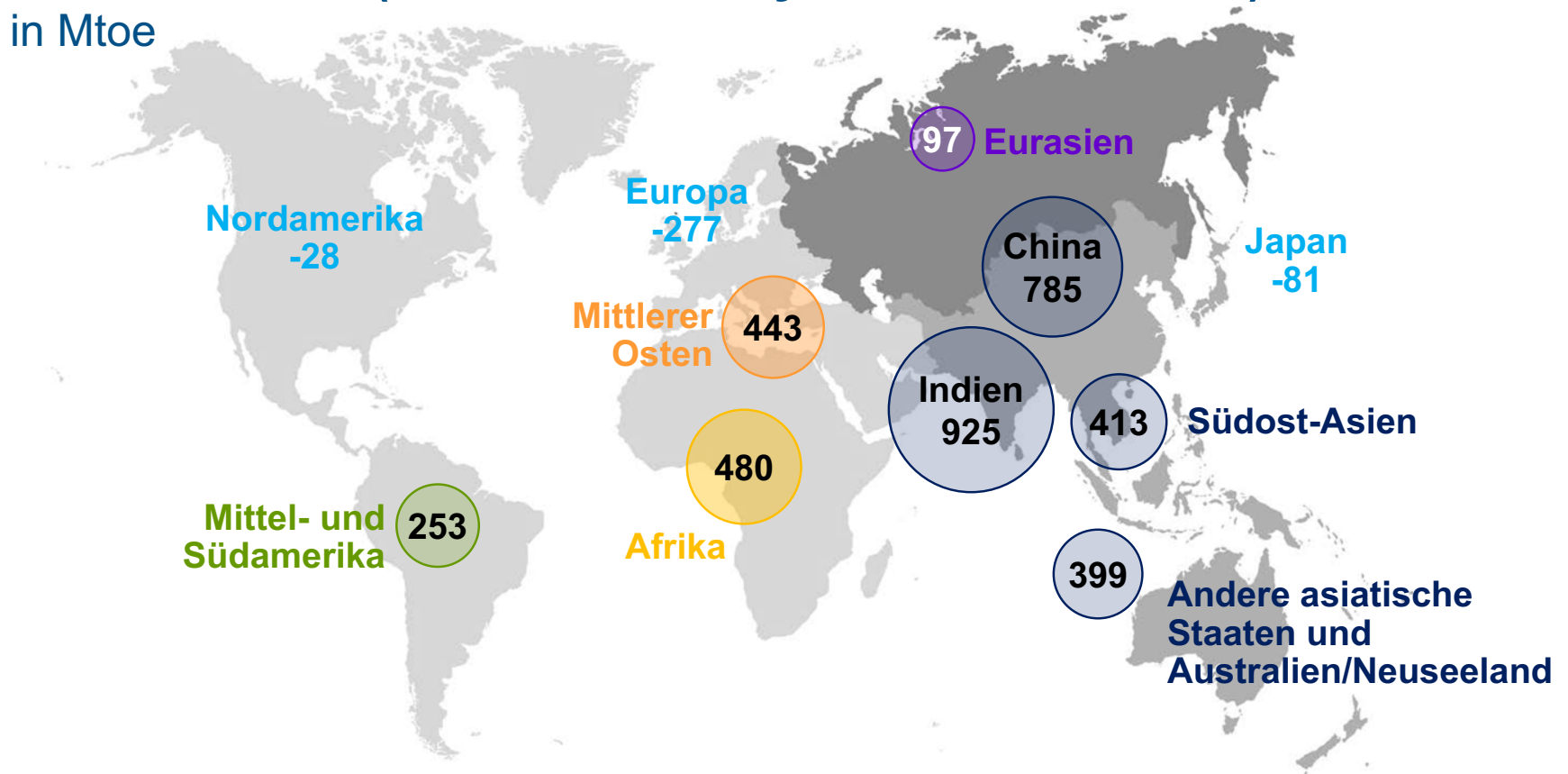

Abb. 14 Zuwachs im Primärenergieverbrauch nach Weltregionen 2018 bis 2040 (im Stated Policy Scenario der IEA) - in Mtoe. Wachstum konzentriert sich auf Asien, den Mittleren Osten, Afrika und Südamerika. Rückgang in Europa, Nordamerika und Japan. Quelle: IEA, World Energy Outlook (2019)

sich gemäß der WEC-Studie die Stromerzeugung auf Basis Solarenergie im Szenario Hard Rock verfünfzehnfachen, in Modern Jazz mehr als verdreißigfachen und in Unfinished Symphony sogar mehr als verfünzigfachen - jeweils im Vergleich zum Stand des Jahres 2015. Je nach Szenario lösen Sonne bzw. Wind in den nächsten Jahrzehnten die Wasserkraft als global bisher wichtigste erneuerbare Energiequelle zur Stromerzeugung ab. Gleichwohl gehört die Wasserkraft auch künftig zu den Großen Drei unter den erneuerbaren Energien zur Stromversorgung (Abb. 10).

Entscheidende Gründe für den starken Zuwachs bei Wind und Solar sind - neben der in vielen Staaten praktizierten staatlichen Förderung zugunsten dieser Technologien - die stark gesunkenen Erzeugungskosten (International Renewable Energy Agency 2020) (Tab. 1).

Wie drastisch sich der Einsatzmix zur Stromerzeugung künftig ändert, wird für den Zeitraum bis 2040 in Abb. 11 und für den Zeitraum bis 2060 in Abb. 12 veranschaulicht. Es wird deutlich, dass der gesamte Zuwachs der Stromerzeugung durch erneuerbare Energien gedeckt werden wird. In den normativen Szenarien erfolgt sogar bereits bis 2040 zusätzlich eine signifikante Substitution von fossilen durch erneuerbare Energien - vor allem zu Lasten der Kohle. So halten beispielsweise im Sustainable Development Scenario der IEA die fossilen Energien im Jahr 2040 nur noch einen Anteil von zusammen $21 \%$ an der globalen Stromerzeugung. Für Kernenergie kommen die Modellrechnungen in diesem Zielszenario für 2040 auf einen Anteil von $11 \%$ und für die erneuerbaren Energien von $68 \%$.

Schlüsselbotschaft Während der Anteil erneuerbarer Energien an der weltweiten Stromerzeugung zwischen 1970 und 2015 konstant geblieben war, zeichnet sich für die kommenden Jahrzehnte ein bisher nie dagewesenes Wachstum ab. Dieser Paradigmenwechsel katapultiert die erneuerbaren Energien künftig auf Rang 1 unter den zur Stromerzeugung genutzten Energien.

\subsection{Verschiebung der Verbrauchsschwerpunkte nach Weltregionen}

Der Zuwachs, der künftig noch beim Primärenergieverbrauch erwartet wird, spielt sich vor allem in den Entwicklungs- und Schwellenländern ab. Während in den OECDStaaten mit einer Stagnation bzw. sogar einem Rückgang des Verbrauchs zu rechnen ist, wird für die Nicht-OECDStaaten mit einem Anstieg gerechnet, auch wenn dieser verhaltener ausfallen dürfte als in der Vergangenheit (Abb. 13).

Damit verbunden ist auch eine Schwerpunktverlagerung im Energieverbrauch nach Weltregionen. Zuwächse im Ver- 


\section{Verbrauch an Kohle nach Staaten/Regionen im Stated Policies Scenario in Mtce}

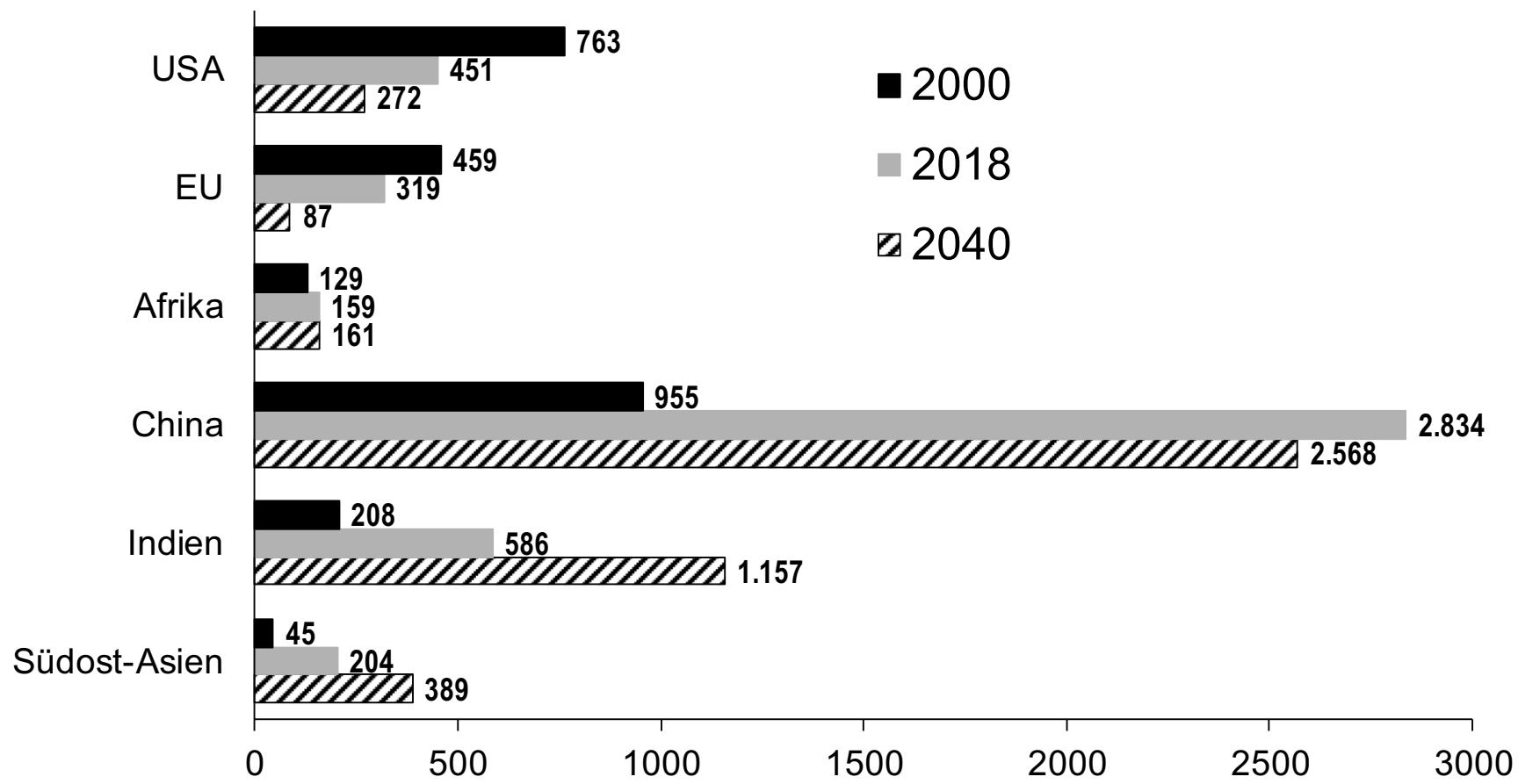

Abb. 15 Verbrauch an Kohle nach Staaten/Regionen im Stated Policies Scenario - in Mtce. Quelle: IEA, World Energy Outlook (2019), Paris 2019

brauch werden insbesondere in den asiatischen Entwicklungs- und Schwellenländern, im Mittleren Osten, in Afrika und auch in Südamerika erwartet. Dagegen ist für Nordamerika, für Europa und für Japan bis 2040 mit einer nachgebenden Nachfrage zu rechnen (Abb. 14).

Besonders drastische Verbrauchseinbußen zeigen sich für Kohle. Dies gilt für Nordamerika, Europa und Japan. Demgegenüber wird ein Wachstum im Kohleverbrauch im Wesentlichen auf verschiedene Staaten Südost-Asiens, vor allem Indien, begrenzt bleiben. In China ist nach einer seit dem Jahr 2000 verzeichneten massiven Erhöhung des Kohleverbrauchs künftig mit einem Rückgang zu rechnen. Trotzdem bleibt die Kohle in China - insbesondere in der Stromerzeugung - auch in den bevorstehenden Jahrzehnten noch eine tragende Säule der Energieversorgung (Abb. 15).

Schlüsselbotschaft Es findet künftig eine starke Verlagerung des Energieverbrauchs Richtung Asien statt.

\subsection{Veränderung im Energieverbrauch nach Sektoren sowie Rolle der Digitalisierung}

Die Versorgungsstrukturen in den verschiedenen volkswirtschaftlichen Sektoren, wie Industrie, Gebäude und Verkehr (Abb. 16), werden sich künftig stärker ändern als in der Vergangenheit. Es steht eine digitale Transformation des Energiesystems bevor. Die Anzahl von Akteuren erhöht sich. Aggregatoren und Prosumer gewinnen an Bedeutung. Es eröffnen sich neue Geschäfts- und Wertschöpfungsmodelle. Intelligente Technologien verändern nicht nur die Art und Weise, wie Strom produziert wird sondern beeinflussen auch die Übertragung und Verteilung an Verbraucher sowie die Integration von Strom aus Anlagen der Verbraucher in die Netze bis hin zu den Abrechnungsprozessen.

Die Stromnetze werden massiv verstärkt und erweitert, um den künftigen Anforderungen gerecht zu werden. DNVGL beziffert die weltweiten Stromnetzausgaben im Jahr 2050 auf etwa 1750 Mrd. US\$. Das entspricht mehr als einer Verdopplung im Vergleich zum gegenwärtigen Stand von jährlich etwa $750 \mathrm{Mrd}$. US\$. Das künftige Netz wird nicht nur wegen der Erweiterungsinvestitionen zur Integration der erneuerbaren Energien und wegen der steigenden 


\section{Primärenergieverbrauch nach Endverbrauchssektoren ${ }^{a}$ - gemäß ET-Szenario im 2019 BP Energy Outlook in Milliarden Tonnen Öläquivalent (Mrd. toe)}

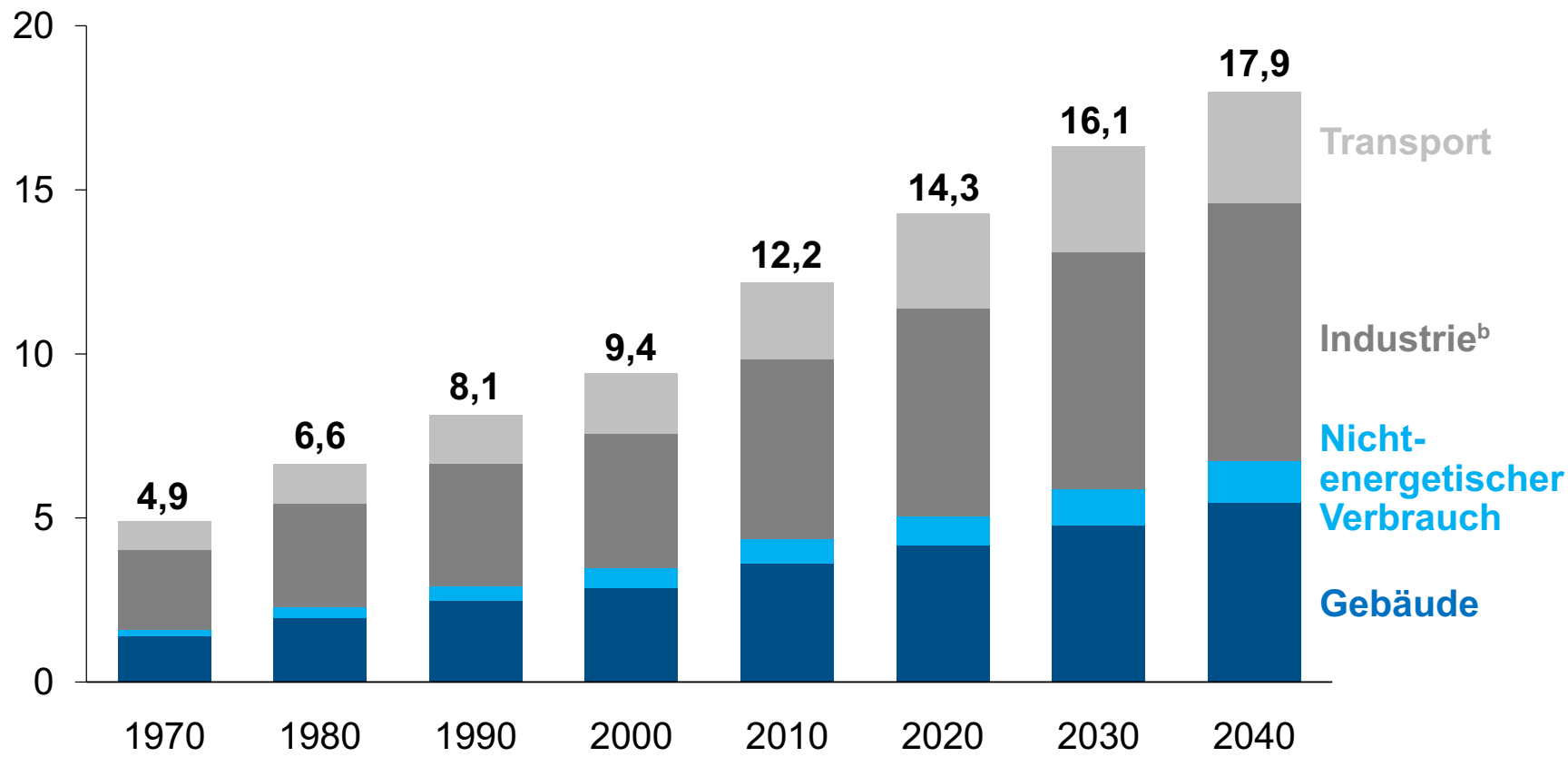

Abb. 16 Primärenergieverbrauch nach Endverbrauchssektoren ${ }^{\mathrm{a}}$ - gemäß ET-Szenario im 2019 BP Energy Outlook - in Milliarden Tonnen Öläquivalent (Mrd. toe). ${ }^{2}$ Der Primärenergieverbrauch zur Stromerzeugung ist den Endverbrauchssektoren gemäß deren Stromverbrauch zugeordnet. bIndustrie ohne nichtenergetischen Verbrauch. Quelle. 2019 BP Energy Outlook, Outlook for Energy: A Perspective to 2040

Strom-Nachfrage erheblich größer sondern auch effizienter - von künstlicher Intelligenz mittels Datenplattformen gesteuert.

Digitale Informations- und Kommunikationstechnologien werden die Vernetzung zwischen den Wertschöpfungsstufen in horizontaler (Sektorenkopplung) und in vertikaler Richtung verstärken. Durch die Erweiterung digitaler Plattformen wird die Transparenz der Märkte für Regulierungsbehörden und andere Stakeholder verbessert. Die Digitalisierung dringt vermehrt in alle Sektoren ein.

- In der industriellen Produktion wird die fortgesetzte Automatisierung begünstigt. Optimierte industrielle Prozesse vermindern den Energieverbrauch und reduzieren den Abfall.

- Im Gebäudebereich finden vermehrt Steuerungstechnologien Eingang, die zur Verbesserung der Energieeffizienz führen und auch die Möglichkeit eröffnen, in $\mathrm{Zu}$ sammenarbeit mit Energieversorgern den Verbrauch nach Maßgabe der jeweiligen Preise zu steuern. Mittels zusätzlicher Speichermöglichkeiten in Gebäuden sind auch Rückführungen von Energie in die Netze möglich.
- Im Individualverkehr spielen Veränderungen in der Antriebsart, Car Sharing und neue Arten des Transports eine wachsende Rolle. Es werden sich Geschäftsmodelle entwickeln, die den Fahrzeugbesitzern Anreize bieten, die Batterien ihrer Elektrofahrzeuge als temporäre Komponenten für den Ausgleich von Angebot und Nachfrage zur Verfügung zu stellen.

In allen drei genannten Sektoren wird die Digitalisierung $\mathrm{zu}$ einer verbesserten Koordination zwischen den Komponenten und damit zu einer Optimierung des Gesamtsystems führen. Dies ist mit einem deutlichen Anstieg des Strombedarfs verknüpft. Damit ist ein erheblicher Dekarbonisierungseffekt verbunden, da Strom zunehmend durch erneuerbare Energien bereit gestellt wird. Eine verstärkte Nutzung von Strom in allen Sektoren wird entsprechend als Schlüsselelement für die Dekarbonisierung gesehen.

Schlüsselbotschaft Digitalisierung, Dezentralisierung und Dekarbonisierung durch Elektrifizierung kennzeichnen die neue Energiewelt. 


\title{
Weltweiter Bestand an PKW nach Antriebstechnologien gemäß den Szenarien des World Energy Council
}

\author{
in Millionen
}

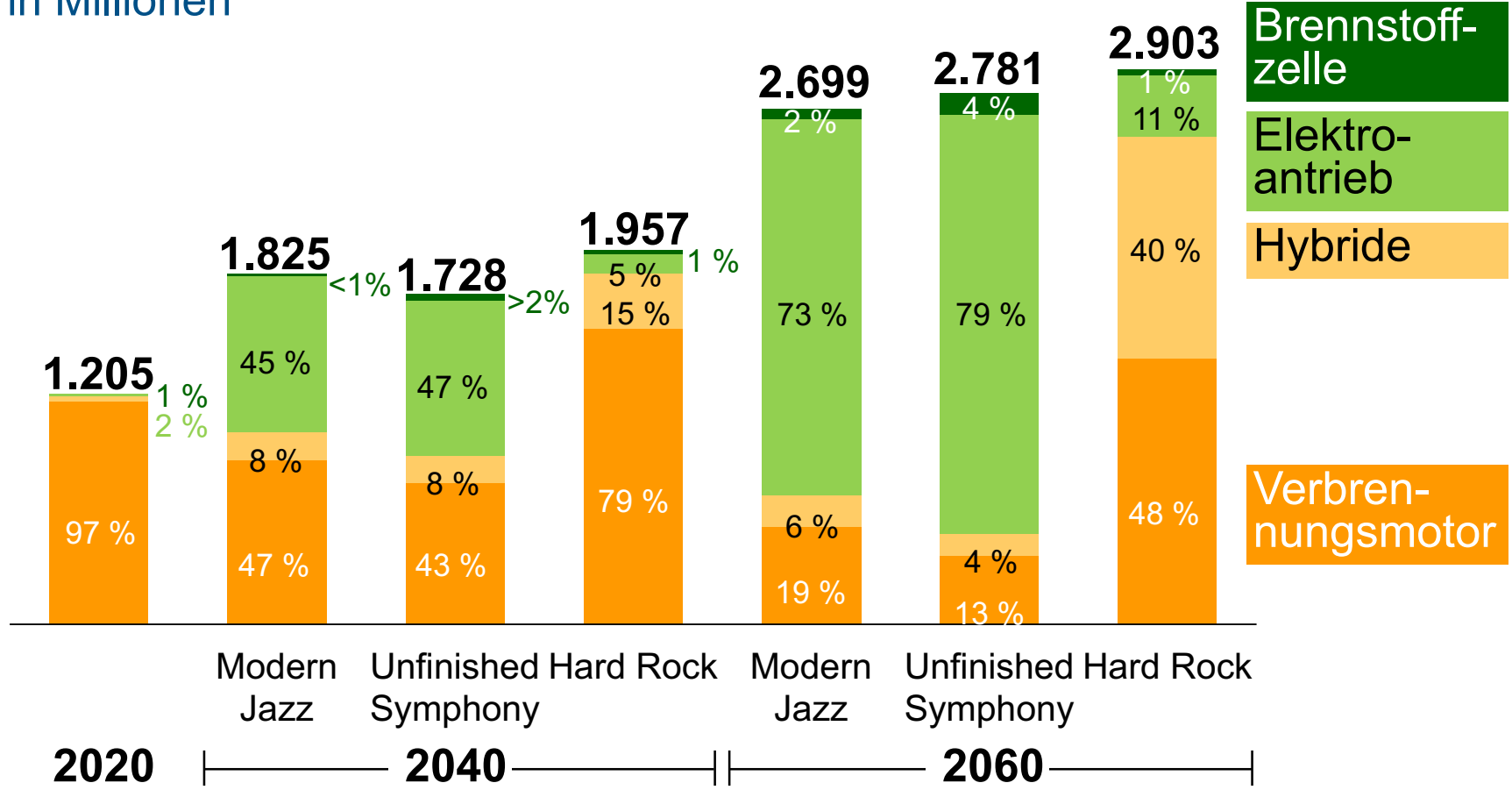

Abb. 17 Weltweiter Bestand an PKW nach Antriebstechnologien gemäß den Szenarien des World Energy Council - in Millionen. Quelle: Paul Scherrer Institut

\subsection{Rolle von Wasserstoff und synthetischen Brennstoffen}

Im Rahmen der Transformation der Energieversorgung wird künftig Wasserstoff eine zentrale Rolle eingeräumt. Wasserstroff kann als wesentliches Element der Sektorenkopplung fungieren. In den Bereichen, in denen Strom aus erneuerbaren Energien nicht direkt eingesetzt werden kann, eröffnen mittels Elektrolyse auf Basis erneuerbarer Energien hergestellter Wasserstoff und seine Folgeprodukte (Power-to-X) zusätzliche Dekarbonisierungspfade.

Der heute fossil erzeugte Wasserstoff, der in chemischen und industriellen Prozessen genutzt wird, etwa zur Herstellung von Ammoniak, kann durch erneuerbar erzeugten Wasserstoff ersetzt werden. Für die treibhausgasneutrale Erzeugung von Primärstahl ist der Einsatz von Wasserstoff als Ersatz für Steinkohlenkoks ein technologisch vielversprechender Pfad.

Im Verkehrssektor kann Wasserstoff zum Beispiel in Brennstoffzellen die $\mathrm{CO}_{2}$-freie Mobilität befördern und als Basis für synthetische Kraftstoffe genutzt werden. Im Luftund Seeverkehr sind für die Dekarbonisierung klimaneutra- le synthetische Brennstoffe erforderlich. Außerdem kann durch die Einführung von Brennstoffzellenfahrzeugen, u.a. im öffentlichen Personennahverkehr (Busse, Züge), in Teilen des Straßenschwerlastverkehrs (LKW) oder in der Logistik, die batterieelektrische Mobilität ergänzt werden. Auch im Gebäudesektor sind synthetische Brennstoffe einsetzbar.

Erneuerbar erzeugter Strom kann zudem angebotsorientiert und flexibel in Wasserstoff umgewandelt werden. Durch die damit eröffnete Speichermöglichkeit kann ein Beitrag zum Ausgleich von Angebot und Nachfrage geleistet werden.

Der World Energy Council sieht die größten Chanen für einen Durchbruch der Wasserstofftechnologie im Szenario Unfinished Symphony, das auf eine starke internationale Kooperation setzt. So kommen die Modellrechnungen des Paul Scherrer Instituts zu dem Ergebnis, dass sich der Anteil von Wasserstoff am globalen Energieverbrauch des Verkehrssektors bis 2060 im Szenario Unfinished Symphony auf $11 \%$ erhöht - gegenüber 6\% im Szenario Modern Jazz und nur $1 \%$ im Szenario Hard Rock. Für den Individualverkehr kommt der WEC zu folgender Einschätzung: Gemäß 


\section{Globale $\mathrm{CO}_{2}$-Emissionen gemäß den Szenarien des World Energy Council \\ in Mrd. $\mathrm{t}$}

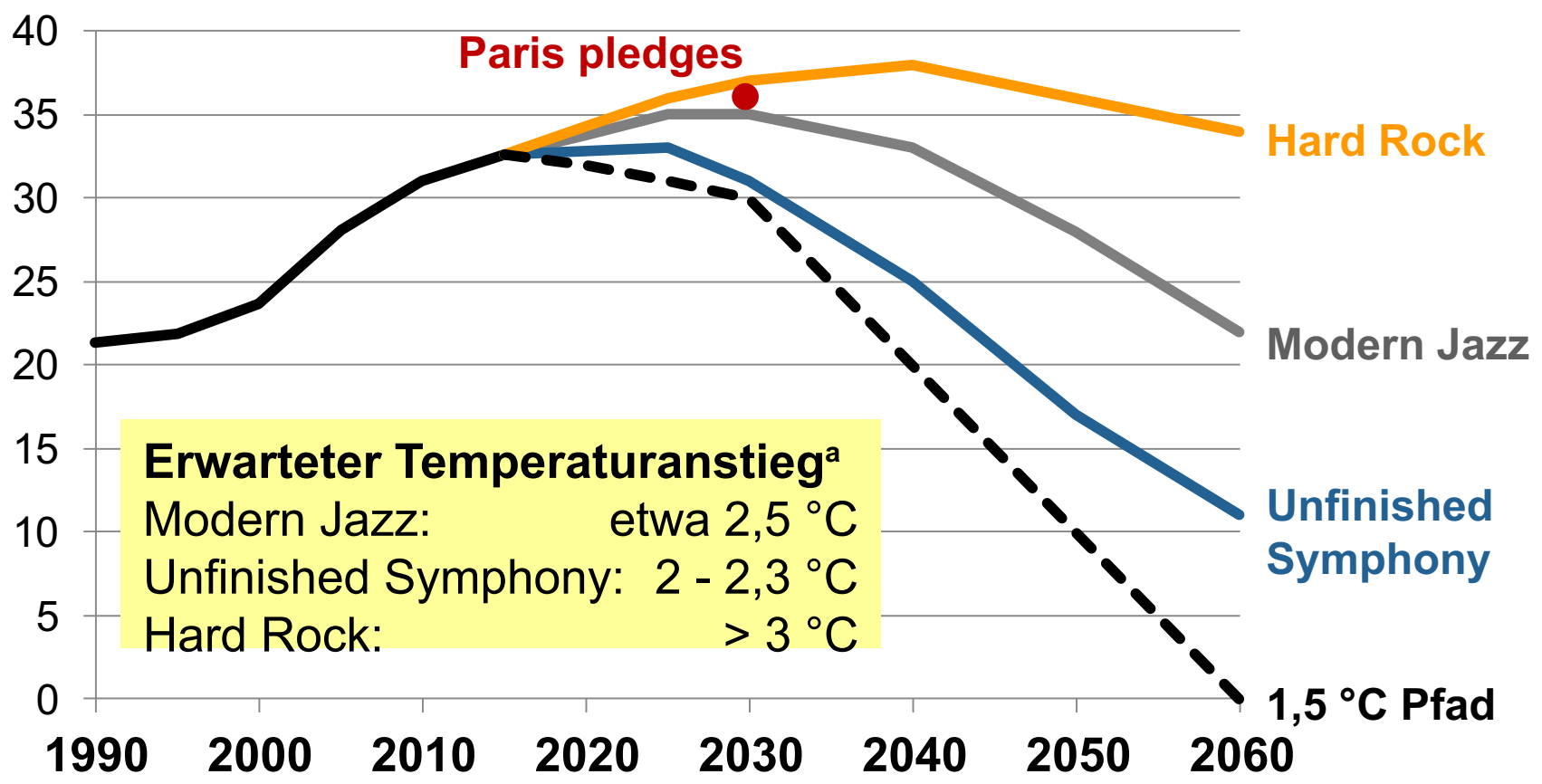

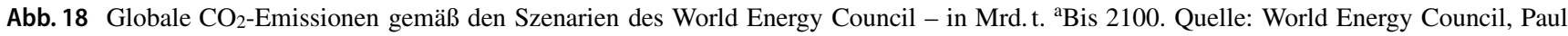
Scherrer Institute, Accenture Strategy World Energy Scenarios (2019b), September 2019

dem Szenario Unfinished Symphony werden im Jahr 2060 weltweit etwa 117 Mio. Pkw entsprechend $4,2 \%$ des weltweiten Bestandes mit Brennstoffzellen betrieben werden gegenüber 66 Mio. im Szenario Modern Jazz entsprechend 2,4\% des PkW-Bestands. Bei Hard Rock wird demgegenüber kein echter Durchbruch der Wasserstofftechnologie gesehen (Abb. 17). Insgesamt führen die den WEC-Szenarien zugrunde liegenden Modellrechnungen zu dem Ergebnis, dass sich der weltweite Bestand an Pkw von etwa 1,2 Mrd. im Jahr 2020 auf 2,7 Mrd. im Szenario Modern Jazz, auf 2,8 Mrd. im Szenario Unfinished Symphony und auf 2,9 Mrd. im Szenario Hard Rock im Jahr 2060 mehr als verdoppelt. Dies wird mit der erhöhten Motorisierung in Südamerika, in Afrika sowie in den Entwicklungs- und Schwellenländern Asiens erklärt. In den Szenarien Unfinished Symphony und Modern Jazz wird erwartet, dass 2060 etwa drei Viertel des globalen Pkw-Bestandes vollelektrisch betrieben werden.

Schlüsselbotschaft Wasserstoff und synthetisch hergestellten Brennstoffen kommt künftig - neben einer verstärkten Elektrifizierung - eine wichtige Rolle zur Realisierung der angestrebten Dekarbonisierung der Energieversorgung zu.

\subsection{Einhaltung der Klimaziele}

In allen vorgelegten Projektionen und exploratorischen Szenarien wird das ambitionierte Ziel des Pariser Klima-Übereinkommens verfehlt. Dies gilt selbst für das WEC-Szenario Unfinished Symphony - trotz der in diesem Szenario unterstellten hohen $\mathrm{CO}_{2}$-Preise und der Annahme, dass sich weltweit ein einheitlicher $\mathrm{CO}_{2}$-Preis von 90 US\$ pro Tonne ab 2050 und von 110 US\$ pro Tonne im Jahr 2060 (ausgedrückt in 2010 US\$) einstellt und ein hohes Maß an internationaler Kooperation die Zusammenarbeit der Staaten bestimmt (Abb. 18).

Nur in den normativen Szenarien wird das 2-Grad-Ziel erreicht, dies aber per Definition. Damit wird deutlich, dass zum Erreichen der Ziele nicht nur die erneuerbaren Energien noch stärker ausgebaut und die Energieeffizienz massiv gesteigert werden müssen, sondern dass auch eine breite Umsetzung der Technologie der Abscheidung und Nutzung bzw. Speicherung von $\mathrm{CO}_{2}$ unverzichtbar ist. Ergänzend dazu werden auch Strategien zur Erzeugung negativer Emissionen als erforderlich angesehen. $\mathrm{Zu}$ den negativen Emissionstechnologien gehören u. a. Aufforstung, Biomasse-Verstromung mit Carbon Capture and Storage, Direct 
Air Carbon Capture and Storage sowie die Förderung von Kohlenstoffbindung im Boden durch Biomassewachstum.

Schlüsselbotschaft Eine Verstärkung der politischen Rahmenbedingungen zugunsten aller $\mathrm{CO}_{2}$-Vermeidungstechnologien, eine umfassende internationale Kooperation und eine globale Bepreisung von $\mathrm{CO}_{2}$ sind notwendig, um den bestehenden Klimazielen gerecht zu werden.

\section{Fazit unter Berücksichtigung von COVID- 19}

Die COVID-19 Pandemie hat zumindest die kurz- und mittelfristigen Einschätzungen zur Entwicklung des globalen Energieverbrauchs und der Treibhausgas-Emissionen signifikant verändert. Erste Analysen - vorgelegt durch die Internationale Energie-Agentur - kommen zu dem Ergebnis, dass sich die globale Energienachfrage 2020 als Folge einer verminderten Wirtschaftsleistung und der verhängten Beschränkungen um mindestens $6 \%$ im Vergleich zum Vorjahr verringern wird (International Energy Agency 2020). Selbst wenn damit die eintretende Entwicklung noch unterschätzt wäre, bedeutete dies den größten Rückgang innerhalb der letzten 70 Jahre in relativen Größen und die stärkste jemals erzielte Reduktion in absoluten Zahlen. Die Auswirkungen der COVID-19-Krise auf die Energienachfrage wird damit noch deutlich größer ausfallen als der Effekt der Finanzkrise, die im Jahr 2009 zu einem Rückgang des weltweiten Energieverbrauchs um 1,5\% im Vergleich zum Jahr 2008 geführt hatte.

Die einzelnen Energieträger sind von der Entwicklung, die sich für 2020 abzeichnet, unterschiedlich betroffen. Für Kohle und Öl werden Verbrauchsminderungen zwischen 8 und $10 \%$ erwartet. Die Nachfrage nach Erdgas dürfte ebenfalls deutlich niedriger als 2019 ausfallen; Gründe sind reduzierte Einsätze zur Stromerzeugung und in Industrieprozessen. Die Einbußen bei Kernenergie werden demgegenüber deutlich geringer eingeschätzt. Bei erneuerbaren Energien dürfte zwar die im Vergleich zum Vorjahr verringerte Installation neuer Anlagen den Wachstumstrend ausbremsen; aufgrund des Einspeisevorrangs in der Stromerzeugung wird aber mit einer Konstanz des Verbrauchs im Vergleich zu 2019 gerechnet. Die $\mathrm{CO}_{2}$-Emissionen werden 2020 deutlich sinken und den niedrigsten Stand seit dem Jahr 2010 erreichen.

Vor dem Hintergrund dieser aktuellen Entwicklung stellt sich die Frage, welche Auswirkungen auf die Höhe und die Struktur des Energieverbrauchs sowie die Entwicklung der Treibhausgas-Emissionen mittel- und längerfristig zu erwarten sind. Dies wird Gegenstand künftiger Analysen sein, die beispielsweise im September 2020 von BP und von der U.S. Energy Information Administration sowie im November 2020 von der International Energy Agency mit dem World Energy Outlook 2020 vorgelegt werden. Aber bereits jetzt lässt sich sagen, dass der globale Energieverbrauch niedriger ausfallen dürfte, als bisher angenommen, die Elektrifizierung einen zusätzlichen Schub bekommt, Wasserstoff und synthetischen Brennstoffen durch entsprechend gestaltete politische Strategien vermehrt zum Durchbruch verholfen wird und die Entwicklung der Treibhausgas-Emissionen gedämpft werden wird. Auch hat sich das politische Augenmerk in jüngster Zeit vermehrt auf negative Emissionstechnologien gerichtet, die als Schlüssel für den Weg in die bis zur Mitte dieses Jahrhunderts angestrebte Treibhausgas-Neutralität der Energieversorgung gesehen wird (Weltenergierat - Deutschland 2020).

\section{Literatur}

BP (2019) Energy outlook to 2040. London. https://www.bp.com/ content/dam/bp/business-sites/en/global/corporate/pdfs/energyeconomics/energy-outlook/bp-energy-outlook-2019.pdf. Zugegriffen: 15. Juni 2020

DNV-GL (2019) Energy transition outlook to 2050. Oslo. https://www. dnvgl.com/publications/energy-transition-outlook-2019-162874. Zugegriffen: 15. Juni 2020

Energy Information Administration (2019) International energy outlook 2019. Washington DC. https://www.eia.gov/outlooks/ieo/. Zugegriffen: 15. Juni 2020

Equinor (2019) Energy perspectives to 2050. Stavanger. https://www. equinor.com/content/dam/statoil/documents/energy-perspectives/ Energy\%20Perspectives\%202019\%20report.pdf. Zugegriffen: 15. Juni 2020

ExxonMobil (2019) 2019 outlook for energy: a perspective to 2040. Irving. https://corporate.exxonmobil.com/Energy-and-environment/ Looking-forward/Outlook-for-Energy. Zugegriffen: 20. Juni 2020

International Energy Agency (2019) World energy outlook 2019. Paris. https://www.iea.org/reports/world-energy-outlook-2019. Zugegriffen: 15. Juni 2020

International Energy Agency (2020) Global energy review 2020-the impacts of the Covid-19 crisis on global energy demand and $\mathrm{CO} 2$ emissions. Paris. https://webstore.iea.org/global-energy-review2020. Zugegriffen: 15. Juni 2020

International Renewable Energy Agency (2020) Renewable power generation costs in 2019. Abu Dhabi. https://www.irena.org/ publications/2020/Jun/Renewable-Power-Costs-in-2019. Zugegriffen: 15. Juni 2020

IRENA (2020) Renewable power generation costs in 2019. International Renewable Energy Agency, Abu Dhabi

McKinsey \& Company (2019) Global energy perspective 2019: reference case. New York City. https://www.mckinsey.com. Zugegriffen: 15. Juni 2020

Shell International B.V. (2018) Shell scenarios: Sky-meeting the goals of the Paris agreement. Den Haag. https://www.shell.com/ skyscenario. Zugegriffen: 15. Juni 2020

Weltenergierat - Deutschland (2020) Energie für Deutschland 2020. Berlin. https://www.weltenergierat.de/publikationen/energiefuer-deutschland/. Zugegriffen: 15. Juni 2020 
World Energy Council (2019a) Innovation insights brief-global energy scenarios comparison review. London. https://www. worldenergy.org/publications/entry/innovation-insights-briefglobal-energy-scenarios-comparison-review. Zugegriffen: 15 . Juni 2020

World Energy Council (2019b) World energy scenarios 2019. London. https://www.worldenergy.org/publications/entry/world-energyscenarios-2019-exploring-innovation-pathways-to-2040. Zugegriffen: 15. Juni 2020 